\title{
Planck early results. XXII. The submillimetre properties of a sample of Galactic cold clumps ${ }^{\star, \star \star}$
}

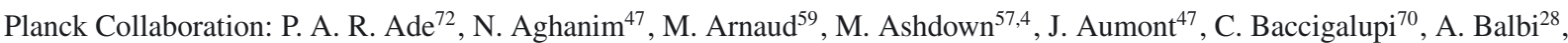
A. J. Banday ${ }^{76,7,64}$, R. B. Barreiro ${ }^{54}$, J. G. Bartlett ${ }^{3,55}$, E. Battaner ${ }^{78}$, K. Benabed ${ }^{48}$, A. Benôt ${ }^{46}$, J.-P. Bernard ${ }^{76,7}$, M. Bersanelli ${ }^{25,41}$, R. Bhatia ${ }^{5}$, J. J. Bock ${ }^{55,8}$, A. Bonaldi ${ }^{37}$, J. R. Bond ${ }^{6}$, J. Borrill ${ }^{63,73}$, F. R. Bouchet ${ }^{48}$, F. Boulanger ${ }^{47}$, M. Bucher ${ }^{3}$, C. Burigana ${ }^{40}$, P. Cabella ${ }^{28}$, C. M. Cantalupo ${ }^{63}$, J.-F. Cardoso ${ }^{60,3,48}$, A. Catalano ${ }^{3,58}$, L. Cayón ${ }^{18}$, A. Challinor ${ }^{50,57,9}$, A. Chamballu ${ }^{44}$, L.-Y Chiang ${ }^{49}$, P. R. Christensen ${ }^{67,29}$, D. L. Clements ${ }^{44}$, S. Colombi ${ }^{48}$, F. Couchot ${ }^{62}$, A. Coulais ${ }^{58}$, B. P. Crill ${ }^{55,68}$, F. Cuttaia ${ }^{40}$, L. Danese ${ }^{70}$, R. D. Davies ${ }^{56}$, P. de Bernardis ${ }^{24}$, G. de Gasperis $^{28}$, A. de Rosa ${ }^{40}$, G. de Zotti ${ }^{37,70}$, J. Delabrouille ${ }^{3}$, J.-M. Delouis ${ }^{48}$, F.-X. Désert ${ }^{43}$, C. Dickinson ${ }^{56}$, Y. Doi ${ }^{14}$, S. Donzelli ${ }^{41,52}$, O. Doré ${ }^{55,8}$, U. Dörl ${ }^{64}$, M. Douspis ${ }^{47}$, X. Dupac ${ }^{33}$, G. Efstathiou ${ }^{50}$, T. A. Enßlin ${ }^{64}$, E. Falgarone ${ }^{58}$, F. Finelli ${ }^{40}$, O. Forni ${ }^{76,7}$, M. Frailis ${ }^{39}$, E. Franceschi ${ }^{40}$,

S. Galeotta ${ }^{39}$, K. Ganga ${ }^{3,45}$, M. Giard ${ }^{76,7}$, G. Giardino ${ }^{34}$, Y. Giraud-Héraud ${ }^{3}$, J. González-Nuevo ${ }^{70}$, K. M. Górski ${ }^{55,80}$, S. Gratton ${ }^{57,50}$ A. Gregorio ${ }^{26}$, A. Gruppuso ${ }^{40}$, F. K. Hansen ${ }^{52}$, D. Harrison ${ }^{50,57}$, G. Helou ${ }^{8}$, S. Henrot-Versilléé ${ }^{62}$, D. Herranz ${ }^{54}$, S. R. Hildebrandt ${ }^{8,61,53}$, E. Hivon ${ }^{48}$, M. Hobson ${ }^{4}$, W. A. Holmes ${ }^{55}$, W. Hovest ${ }^{64}$, R. J. Hoyland ${ }^{53}$, K. M. Huffenberger ${ }^{79}$, N. Ikeda ${ }^{51}$, A. H. Jaffe ${ }^{44}$, W. C. Jones ${ }^{17}$, M. Juvela ${ }^{16}$, E. Keihänen ${ }^{16}$, R. Keskitalo ${ }^{55,16}$, T. S. Kisner ${ }^{63}$, Y. Kitamura ${ }^{51}$, R. Kneissi ${ }^{32,5}$, L. Knox ${ }^{20}$, H. Kurki-Suonio ${ }^{16,35}$, G. Lagache ${ }^{47}$, J.-M. Lamarre ${ }^{58}$, A. Lasenby ${ }^{4,57}$, R. J. Laureijs ${ }^{34}$, C. R. Lawrence ${ }^{55}$, S. Leach ${ }^{70}$, R. Leonardi ${ }^{33,34,21}$, C. Leroy ${ }^{47,76,7}$, M. Linden-Vørnle ${ }^{11}$, M. López-Caniego ${ }^{54}$, P. M. Lubin ${ }^{21}$, J. F. Macías-Pérez ${ }^{61}$, C. J. MacTavish ${ }^{57}$, B. Maffei ${ }^{56}$, J. Malinen ${ }^{16}$, N. Mandolesi ${ }^{40}$, R. Mann ${ }^{71}$, M. Maris ${ }^{39}$, D. J. Marshall ${ }^{76,7}$, P. Martin ${ }^{6}$, E. Martínez-González ${ }^{54}$, S. Masi $^{24}$, S. Matarrese ${ }^{23}$, F. Matthai ${ }^{64}$, P. Mazzotta ${ }^{28}$, P. McGehee ${ }^{45}$, A. Melchiorri ${ }^{24}$, L. Mendes ${ }^{33}$, A. Mennella ${ }^{25,39}$, C. Meny ${ }^{76,7}$, S. Mitra ${ }^{55}$, M.-A. Miville-Deschênes ${ }^{47,6}$, A. Moneti ${ }^{48}$, L. Montier ${ }^{76,7}$, G. Morgante ${ }^{40}$, D. Mortlock ${ }^{44}$, D. Munshi ${ }^{72,50}$, A. Murphy ${ }^{66}$, P. Naselsky ${ }^{67,29}$, F. Nati ${ }^{24}$, P. Natoli ${ }^{27,2,40}$, C. B. Netterfield ${ }^{13}$, H. U. Nørgaard-Nielsen ${ }^{11}$, F. Noviello ${ }^{47}$,

D. Novikov $^{44}$, I. Novikov ${ }^{67}$, S. Osborne ${ }^{75}$, L. Pagani ${ }^{58}$, F. Pajot ${ }^{47}$, R. Paladini ${ }^{74,8}$, F. Pasian ${ }^{39}$, G. Patanchon ${ }^{3}$, V.-M. Pelkonen ${ }^{45}$, O. Perdereau ${ }^{62}$, L. Perotto ${ }^{61}$, F. Perrotta ${ }^{70}$, F. Piacentini ${ }^{24}$, M. Piat ${ }^{3}$, S. Plaszczynski ${ }^{62}$, E. Pointecouteau ${ }^{76,7}$, G. Polenta ${ }^{2,38}$, N. Ponthieu ${ }^{47}$, T. Poutanen ${ }^{35,16,1}$, G. Prézeau ${ }^{8,55}$, S. Prunet ${ }^{48}$, J.-L. Puget ${ }^{47}$, W. T. Reach ${ }^{77}$, R. Rebolo ${ }^{53,30}$, M. Reinecke ${ }^{64}$, C. Renault ${ }^{61}$, S. Ricciardi ${ }^{40}$, T. Riller ${ }^{64}$, I. Ristorcelli $^{76,7}$, G. Rocha ${ }^{55,8}$, C. Rosset ${ }^{3}$, M. Rowan-Robinson ${ }^{44}$, J. A. Rubiño-Martín ${ }^{53,30}$, B. Rusholme ${ }^{45}$, M. Sandri ${ }^{40}$, D. Santos ${ }^{61}$, G. Savini ${ }^{69}$, D. Scott ${ }^{15}$, M. D. Seiffert ${ }^{55,8}$, G. F. Smoot ${ }^{19,63,3}$, J.-L. Starck ${ }^{59,10}$, F. Stivoli ${ }^{42}$, V. Stolyarov ${ }^{4}$, R. Sudiwala ${ }^{72}$, J.-F. Sygnet ${ }^{48}$, J. A. Tauber ${ }^{34}$, L. Terenzi $^{40}$, L. Toffolatti ${ }^{12}$, M. Tomasi ${ }^{25,41}$, J.-P. Torre ${ }^{47}$, V. Toth ${ }^{31}$, M. Tristram ${ }^{62}$, J. Tuovinen ${ }^{65}$, G. Umana ${ }^{36}$, L. Valenziano ${ }^{40}$, P. Vielva ${ }^{54}$, F. Villa ${ }^{40}$, N. Vittorio ${ }^{28}$, L. A. Wade ${ }^{55}$, B. D. Wandelt ${ }^{48,22}$, N. Ysard ${ }^{16}$, D. Yvon ${ }^{10}$, A. Zacchei ${ }^{39}$, and A. Zonca ${ }^{21}$

(Affiliations can be found after the references)

Received 9 January 2011 / Accepted 22 June 2011

\begin{abstract}
We perform a detailed investigation of sources from the Cold Cores Catalogue of Planck Objects (C3PO). Our goal is to probe the reliability of the detections, validate the separation between warm and cold dust emission components, provide the first glimpse at the nature, internal morphology and physical characterictics of the Planck-detected sources. We focus on a sub-sample of ten sources from the C3PO list, selected to sample different environments, from high latitude cirrus to nearby $(150 \mathrm{pc})$ and remote $(2 \mathrm{kpc})$ molecular complexes. We present Planck surface brightness maps and derive the dust temperature, emissivity spectral index, and column densities of the fields. With the help of higher resolution Herschel and AKARI continuum observations and molecular line data, we investigate the morphology of the sources and the properties of the substructures at scales below the Planck beam size. The cold clumps detected by Planck are found to be located on large-scale filamentary (or cometary) structures that extend up to $20 \mathrm{pc}$ in the remote sources. The thickness of these filaments ranges between 0.3 and 3 pc, for column densities $N_{\mathrm{H}_{2}} \sim 0.1$ to $1.6 \times 10^{22} \mathrm{~cm}^{-2}$, and with linear mass density covering a broad range, between 15 and $400 M_{\odot} \mathrm{pc}^{-1}$. The dust temperatures are low (between 10 and $15 \mathrm{~K}$ ) and the Planck cold clumps correspond to local minima of the line-of-sight averaged dust temperature in these fields. These low temperatures are confirmed when AKARI and Herschel data are added to the spectral energy distributions. Herschel data reveal a wealth of substructure within the Planck cold clumps. In all cases (except two sources harbouring young stellar objects), the substructures are found to be colder, with temperatures as low as $7 \mathrm{~K}$. Molecular line observations provide gas column densities which are consistent with those inferred from the dust. The linewidths are all supra-thermal, providing large virial linear mass densities in the range 10 to $300 M_{\odot} \mathrm{pc}^{-1}, \mathrm{comparable}$ within factors of a few, to the gas linear mass densities. The analysis of this small set of cold clumps already probes a broad variety of structures in the C3PO sample, probably associated with different evolutionary stages, from cold and starless clumps, to young protostellar objects still embedded in their cold surrounding cloud. Because of the all-sky coverage and its sensitivity, Planck is able to detect and locate the coldest spots in massive elongated structures that may be the long-searched for progenitors of stellar clusters.
\end{abstract}

Key words. ISM: clouds - dust, extinction - stars: formation - ISM: structure - submillimeter: ISM - stars: protostars

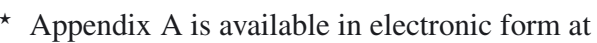
http://www . aanda.org

$\star \star$ Corresponding author: I. Ristorcelli,

e-mail: isabelle.ristorcelli@irap.omp.eu
} 


\section{Introduction}

The main difficulty in understanding star formation lies in the vast range of scales involved in the process, including not only the gravitationally unstable densest and coldest structures, but also their large-scale environment. The characteristics of star formation, described by the stellar mass distribution, the formation efficiency, the evolutionary timescales, and by the modes of formation (clustered or isolated, spontaneous or triggered) are linked to the properties of the cold cores and those of their Galactic surroundings. Theory predicts that the initial mass function (IMF) is largely determined during the pre-stellar core fragmentation phase, but the latter depends on the properties of the pre-stellar cores, particularly the nature of their support against self-gravity, and their density and temperature distributions. Understanding star formation therefore also requires an understanding of the formation and evolution of dense cores. Turbulence, magnetic fields, and gravity all contribute at subparsec scales as well as Galactic scales (see reviews of Bergin \& Tafalla 2007; McKee \& Ostriker 2007; Ward-Thompson et al. 2007; Crutcher et al. 2009).

So far, ground-based (e.g., Motte et al. 1998; Johnstone et al. 2000) and Herschel (e.g., André et al. 2010; di Francesco et al. 2010; Könyves et al. 2010) observations of nearby star-forming regions have revealed a core mass spectrum with a slope similar to that of the IMF. This intriguing result calls for broader investigations, in particular towards the inner Galaxy, where the Galactic environment differs from that of the Solar neighbourhood, and the star formation efficiency is anticipated to be different.

The physical properties of cold cores are still poorly known, possibly because of the short duration of this phase, but mainly because of observational difficulties. The thermal emission of cold dust, intrinsically weak and blended with that of warmer components, must be sought for in the submillimetre range. A combination of continuum and molecular line studies is also needed to develop a global view of the clouds, from large scales (tens of parsecs) down to the scale of dense cores. During the past decade, the development of sensitive continuum and heterodyne detectors at millimetre and submillimetre wavelengths has significantly increased our knowledge of the properties of the cold dark clouds (see Sect. 1 in Planck Collaboration 2011s, hereafter Paper I). The Planck ${ }^{1}$ and Herschel satellites now provide a unique opportunity for studying Galactic dust emission and in particular its dense and cold component. The first Herschel surveys have already provided new insights into cold cores, although limited to specific fields (André et al. 2010; Bontemps et al. 2010; Könyves et al. 2010; Men'shchikov et al. 2010; Molinari et al. 2010; Motte et al. 2010; Peretto et al. 2010; Schneider et al. 2010; Stutz et al. 2010; Ward-Thompson et al. 2010; Zavagno et al. 2010).

The Planck satellite (Tauber et al. 2010; Planck Collaboration 2011a) provides complementarity to Herschel by carrying out an all-sky survey that is well suited for the systematic detection of cold cores. In Paper I, we present the first statistical results of the cores from this survey. Combining Planck and IRAS $100 \mu \mathrm{m}$ data, we have built a preliminary catalogue of 10,783 cold cores (the Cold Core Catalogue of Planck

\footnotetext{
1 Planck (http://WwW.esa.int/Planck) is a project of the European Space Agency (ESA) with instruments provided by two scientific consortia funded by ESA member states (in particular the lead countries France and Italy), with contributions from NASA (USA) and telescope reflectors provided by a collaboration between ESA and a scientific consortium led and funded by Denmark.
}

Objects, C3PO). A sub-sample of the most reliable detections is delivered as part of the Planck Early Release Compact Source Catalogue (ERCSC, see Planck Collaboration 2011c), i.e. the Early Cold Cores (ECC) catalogue. The cores from C3PO cover a wide range in properties, with: temperature, from $7 \mathrm{~K}$ to $19 \mathrm{~K}$, with a peak around $13 \mathrm{~K}$; density from $10^{2} \mathrm{~cm}^{-3}$ to $10^{5} \mathrm{~cm}^{-3}$, with an average value of $2 \times 10^{3} \mathrm{~cm}^{-3}$; mass from 0.4 to $2.4 \times 10^{5} M_{\odot}$; and size ranging from 0.2 to $18 \mathrm{pc}$. The sources are found to be extended, and more importantly are elongated, with a distribution of aspect ratios extending up to 4 and peaking at twice the beam size of the Planck-HFI instrument. As discussed in Paper I, these properties better match the definition of "clumps" rather than "cores" (see Williams et al. 2000; Bergin \& Tafalla 2007). Clumps may contain dense cores, which are likely the precursors of individual or multiple stars. Thus, the main population seen with Planck does not correspond to single pre-stellar cores, but ensembles of cold substructures. A detailed study of the Planck cold objects requires observations at higher resolution. This is the main objective of the Herschel open time key program "Galactic Cold Cores" that is dedicated to following up of a sample of Planck cores.

In this paper we present the first detailed analysis of ten representative sources from the $\mathrm{C} 3 \mathrm{PO}$ catalogue. The targets have been selected to cover different types of clump, spanning a wide range of mass, temperature, and density, and located in different environments, ranging from high latitude cirrus to nearby and distant molecular complexes. We combine the Planck data with available ancillary data (in particular IRIS, 2MASS and AKARI) and dedicated follow-up observations with Herschel and groundbased radio telescopes. Using these ancillary data, we seek to confirm the Planck detections and to demonstrate the reliability of the method which used only Planck and IRAS data to determine the source parameters that enter the C3PO catalogue (Paper I) and ECC catalogue (Planck Collaboration 2011c,v). The higher resolution continuum and line data make it possible to examine the internal structure of the Planck sources. They provide the first hints about the process of internal fragmentation and the physical state of the compact cores at scales below the size of the Planck beam. These properties are essential for the interpretation of the full $\mathrm{C} 3 \mathrm{PO}$ catalogue and will be a major topic for the projects carrying out follow-up studies of $\mathrm{C} 3 \mathrm{PO}$ and ECC catalogue sources.

After describing the observational data set and the source sample selection (Sect. 2), we explain the analysis methods used to derive physical properties for the cores (Sect. 3). The main results are presented in Sect. 4. By fitting spectral energy distributions (SEDs) we derive temperatures, emissivity spectral indices, and column densities for the cores and the surrounding fields, and estimate the linear mass densities, masses and bolometric luminosities of the cores (Sect. 4.1). The small-scale structures within the Planck clumps are studied with the help of Herschel and AKARI data (Sect. 4.3.1), and the gas properties of a few cores are derived from molecular line data (Sect. 4.3.3). Following a discussion of the physical characteristics of the Planck cores, we present our summary and perspectives for the future in Sect. 6.

\section{Observations}

\subsection{The sources}

We have selected from the C3PO ten sources with high reliability, signal-to-noise ratio $S / N \geq 8$, and low colour temperature, $T \leq 14 \mathrm{~K}$ (see Paper I). An initial Monte Carlo sampling of 
the full C3PO catalogue was performed to prepare a candidate list that covered the full range in Galactic position, temperature, flux, and column density. Further selection was made by examining the Planck data and ancillary information from IRIS (Miville-Deschênes \& Lagache 2005), 2MASS extinction (Skrutskie et al. 2006), and CO line data from Dame et al. (2001) and NANTEN surveys (e.g., Fukui et al. 1999), as well as IRAS and AKARI point source catalogues and by cross-checking the sources with the SIMBAD database. The selected sources represent different large-scale morphologies and environments, including filaments, isolated and clustered structures and highlatitude cirrus clouds. One key criterion was the knowledge of the source distance, derived either with an extinction method (Marshall et al. 2006), by association with a known molecular cloud complex, or through a kinematic distance estimate (see Paper I). Nine out of the ten sources have already been observed with Herschel as part of the open time key program "Galactic cold cores." In particular, the sample includes the three targets observed during the Herschel science demonstration phase (SDP, see Juvela et al. 2010, 2011). Half of the sources in the sample are in the Planck ECC. This is mainly due to the criteria used to select the most reliable detections from the C3PO full catalogue (see Paper I): with $T<14 \mathrm{~K}$ for the colour temperature corresponding to the aperture photometry SED, and a high signal to noise ratio for the source detection $(S / N>15)$.

The targets are listed in Table 1 and presented in detail in the Appendix. The ten sources that we focus on will hereafter be referred to by the labels $\mathrm{S} 1$ through $\mathrm{S} 10$.

\subsection{Planck data}

Planck (Tauber et al. 2010; Planck Collaboration 2011a) is the third generation space mission to measure the anisotropy of the cosmic microwave background (CMB). It observes the sky in nine frequency bands covering $30-857 \mathrm{GHz}$ with high sensitivity and angular resolution from $31^{\prime}$ to $5^{\prime}$. The Low Frequency Instrument (LFI; Mandolesi et al. 2010; Bersanelli et al. 2010; Mennella et al. 2011) covers the 30, 44, and $70 \mathrm{GHz}$ bands with amplifiers cooled to $20 \mathrm{~K}$. The High Frequency Instrument (HFI; Lamarre et al. 2010; Planck HFI Core Team 2011a) covers the $100,143,217,353,545$, and $857 \mathrm{GHz}$ bands with bolometers cooled to $0.1 \mathrm{~K}$. Polarisation is measured in all but the highest two bands (Leahy et al. 2010; Rosset et al. 2010). A combination of radiative cooling and three mechanical coolers produces the temperatures needed for the detectors and optics (Planck Collaboration 2011b). Two data processing centres (DPCs) check and calibrate the data and make maps of the sky (Planck HFI Core Team 2011b; Zacchei et al. 2011). Planck's sensitivity, angular resolution, and frequency coverage make it a powerful instrument for Galactic and extragalactic astrophysics as well as cosmology. Early astrophysics results are given in Planck Collaboration, 2011h-w.

We use data from the Planck-HFI bands at $857 \mathrm{GHz}$, $545 \mathrm{GHz}$ and $353 \mathrm{GHz}$ that cover the main peak of the cold dust emission. By restricting ourselves to these highest frequencies, we can perform the analysis at the best angular resolution provided by Planck, i.e., $\sim 4.5^{\prime}$ full width at half-maximum (FWHM).

Figure 1 displays the $857 \mathrm{GHz}$ surface brightness maps for each $1^{\circ} \times 1^{\circ}$ field. The cores are located at the centres of the maps and show dust emission that ranges from 10 to $220 \mathrm{MJy} \mathrm{sr}^{-1}$. They are embedded in extended structures whose shape varies from large filaments (S4, S5, S6, S7 and S10) to more isolated and apparently compact morphologies. Most cores are extended and elongated compared to the Planck beam. As discussed in Paper I, the ellipticity and extension of the cores are not biased by the local beam shape. As an illustration, Fig. A.5 shows the comparison between the local Point Spread Function provided by the FEBeCoP tool (Mitra et al. 2011) with the elliptical Gaussian fit of the sub-sample detections. Typical sizes are from 0.2 to $11 \mathrm{pc}$. Some cores (S2, S8, and S9) are located near bright, warmer regions, where the intensities are higher by up to a factor of 10. In a few cases, the environment exhibits sharp edges, at large scale (see in particular S1, S3 and S7).

The full set of HFI maps $\left(1^{\circ} \times 1^{\circ}\right.$ fields from 857 to $\left.100 \mathrm{GHz}\right)$ for each source are shown in Figs. A.1-A.3. These maps have been derived from the HEALPix sky maps (Górski et al. 2005). With the exception of source S2, the cold source at the centre of the maps is usually visible down to $143 \mathrm{GHz}$. At $100 \mathrm{GHz}$ the sources become difficult to detect because of the falling intensity of the dust spectrum. Only the Musca filament is clearly visible even at $100 \mathrm{GHz}$, although the surface brightness of the source is low $\left(\sim 0.15 \mathrm{MJy} \mathrm{sr}^{-1}\right)$.

Comparison with the $100 \mu \mathrm{m}$ maps from IRIS (Miville-Deschênes \& Lagache 2005) confirms the conclusion of previous submillimetre surveys (using PRONAOS, Archeops, BLAST, and ground-based telescopes) that the cold dust emission is not traced by the $100 \mu \mathrm{m}$ data, but must be studied using longer wavelengths. The IRIS maps are dominated by the warmer and more extended structure around the cores. This is the basis of the source detection method CoCoCoDeT (Cold Core Colour Detection Tool), that was described in detail in Montier et al. (2010) and in Paper I (Sect. 2.4). It uses as a template the spectrum of the warm emission component estimated from the IRIS $100 \mu \mathrm{m}$ map. Subtraction of the warm component results in residual maps of the cold emission component from which source fluxes are derived. The detection process is applied independently to the 857, 545, and $353 \mathrm{GHz}$ maps, after smoothing HFI and IRIS data to the same angular resolution of $4.5^{\prime}$. The main steps of the method and the core extraction process are illustrated in the Appendix (in Fig. A.4) for S1. The residual $100 \mu \mathrm{m}$ signal that remains when a background model is subtracted from the IRIS map (see Paper I) is still needed to constrain the temperature of the cold core SEDs, which typically peak in the range $200-300 \mu \mathrm{m}$. The analysis of Sect. 4 will also test the accuracy of this procedure. The coordinates, distances, fluxes densities, and sizes of the selected Planck cores are given in Table 1. The values listed there are taken directly from the C3PO catalogue (see Paper I and Sect. 3.1).

\subsection{Herschel observations}

The Herschel photometric observations were carried out with the PACS and SPIRE instruments (Pilbratt et al. 2010; Poglitsch et al. 2010; Griffin et al. 2010). Three fields, S8, S9, S10 (corresponding to the source names PCC249, PCC288, and PCC550) were observed in November and December 2009 as part of the Herschel SDP. The other fields were observed between July and September 2010. Most observations were performed separately with PACS $(100$ and $160 \mu \mathrm{m})$ and with SPIRE $(250,350$ and $500 \mu \mathrm{m})$ in scan mapping mode. Because of the larger field size, S5 and S9 were observed in parallel mode using both instruments simultaneously. The observations employed two orthogonal scanning directions, except for the PACS observations of PCC288 where three scanning directions were used. The data were reduced with Herschel interactive processing environment (HIPE), using the official pipeline with the addition of 

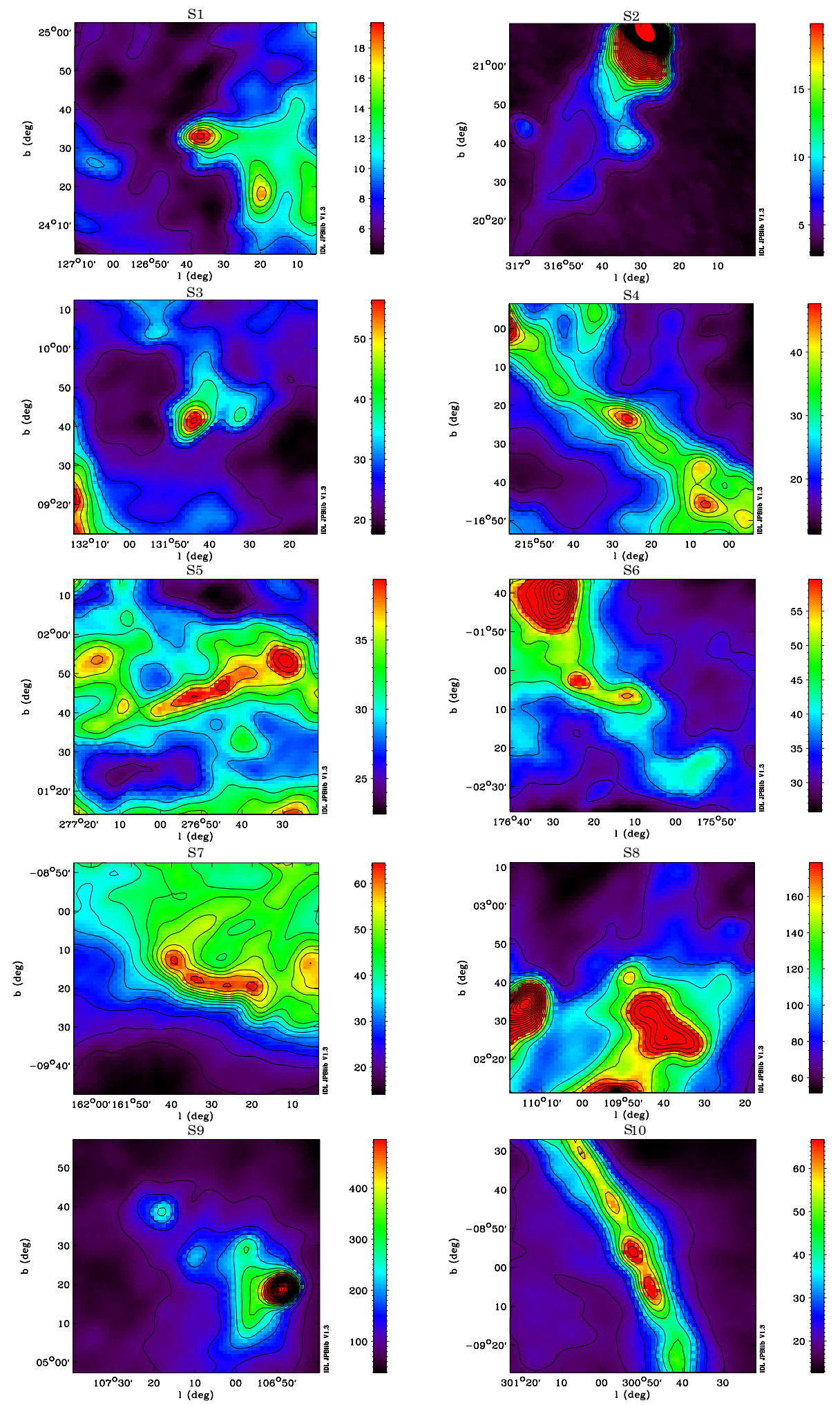

Fig. 1. Planck-HFI brightness emission maps at $857 \mathrm{GHz}$. The colour scale is in $\mathrm{MJy} \mathrm{sr}^{-1}$. Note the large dynamic range of the Planck cold clumps from $10 \mathrm{MJy} \mathrm{sr}^{-1}$ (S2) to $250 \mathrm{MJy} \mathrm{sr}^{-1}$ (S9).

specialised reduction routines to take advantage of the orthogonal scans for deglitching PACS data and to remove SPIRE scan baselines. The PACS maps were created using the MADmap algorithm (Cantalupo et al. 2010). The SPIRE maps are the product of direct projection onto the sky and averaging of the time ordered data, with a baseline correction.

As for most bolometer observations without an absolute calibrator, the zero level (or offset) of the PACS and SPIRE data are arbitrary. We therefore compared the Herschel and PACS data with the predictions of a model constrained by the Planck and IRAS data. The model uses the all-sky dust temperature maps described in Planck Collaboration (2011o) to infer the average radiation field intensity for each pixel at the common resolution level for the Planck and IRAS data. The DUSTEM model (Compiègne et al. 2010) with the above value for the radiation field intensity was used to predict the expected brightness in the Herschel-SPIRE and PACS bands, using the nearest available Planck or IRAS band for normalisation and taking into 
Table 1. Source selection.

\begin{tabular}{|c|c|c|c|c|c|c|c|c|c|c|c|c|}
\hline Source name & & $\begin{array}{c}l \\
(\operatorname{deg})\end{array}$ & $\begin{array}{c}b \\
(\operatorname{deg})\end{array}$ & $S / N$ & $\begin{array}{l}S_{857} \\
(\mathrm{Jy})\end{array}$ & $\begin{array}{l}S_{545} \\
(\mathrm{Jy})\end{array}$ & $\begin{array}{l}S_{353} \\
(\mathrm{Jy})\end{array}$ & $\begin{array}{c}\theta_{\operatorname{Min}}{ }^{a} \\
(\operatorname{arcmin})\end{array}$ & $\begin{array}{c}\theta_{\mathrm{Maj}}^{a} \\
(\operatorname{arcmin})\end{array}$ & $\begin{array}{c}\langle\theta\rangle^{a} \\
(\operatorname{arcmin})\end{array}$ & $\begin{array}{c}d \\
(\mathrm{pc})\end{array}$ & Complex \\
\hline Planck-G126.6+24.5 & S1 & 126.62 & 24.55 & 44.0 & 62.0 & 22.5 & 5.2 & 4.9 & 7.6 & 6.1 & 150 & Polaris flare \\
\hline Planck-G20.7+20.0 & S2 & 316.53 & 20.68 & 20.0 & 35.6 & 12.7 & 2.9 & 7.2 & 9.1 & 8.1 & 550 & Cometary Globule \\
\hline Planck-G131.7+9.7 & S3 & 131.74 & 9.70 & 38.2 & 185.8 & 77.5 & 19.7 & 6.3 & 8.0 & 7.1 & 200 & Cepheus flare \\
\hline Planck-G215.2-16.4 & S4 & 215.44 & -16.38 & 24.0 & 85.9 & 34.9 & 9.2 & 5.0 & 7.4 & 6.1 & 450 & Orion \\
\hline Planck-G276.9+1.7 & S5 & 276.87 & 1.73 & 8.5 & 88.4 & 34.4 & 9.5 & 4.6 & 18.8 & 9.3 & 2000 & Vela \\
\hline Planck-G176.2-2.1 & S6 & 176.18 & -2.11 & 25.8 & 116.8 & 51.0 & 13.2 & 5.0 & 11.5 & 7.6 & 2000 & Perseus Arm \\
\hline Planck-G161.6-9.2 & S7 & 161.56 & -9.29 & 15.0 & 196.5 & 78.1 & 20.9 & 5.5 & 14.9 & 9.1 & 350 & PerOB2 \\
\hline Planck-G109.8+2.7 & S8 & 109.79 & 2.71 & 16.1 & 272.4 & 122.8 & 33.3 & 5.1 & 9.3 & 6.9 & 800 & Cephee \\
\hline Planck-G107.2+5.5 & S9 & 107.17 & 5.45 & 23.6 & 490.2 & 185.5 & 49.5 & 4.4 & 8.3 & 6.1 & 800 & Сер-ОВ3b \\
\hline Planck-G300.9-9.0 & S10 & 300.86 & -8.96 & 82.7 & 267.3 & 98.8 & 24.3 & 4.2 & 15.2 & 8.0 & 225 & Musca \\
\hline
\end{tabular}

Notes. ${ }^{(a)}$ The values are not beam deconvolved; the corresponding ellipse contours are shown in Figs. 3-5.

account the appropriate colour correction in the Herschel filters. The predicted brightness was correlated with the observed maps smoothed to the Planck and IRAS resolution over the region observed with Herschel and the gain and offsets were derived from this correlation. We have used these gain and offset intercalibration values in order to convert the maps from Jy beam ${ }^{-1}$ and $\mathrm{Jy} \mathrm{pix}^{-1}$ (SPIRE and PACS, respectively) into brightness units $\left(\mathrm{MJy} \mathrm{sr}^{-1}\right)$.

\subsection{AKARI observations}

The AKARI satellite (Murakami et al. 2007) has conducted allsky surveys at infrared wavelengths centred at $9 \mu \mathrm{m}, 18 \mu \mathrm{m}$, $65 \mu \mathrm{m}, 90 \mu \mathrm{m}, 140 \mu \mathrm{m}$, and $160 \mu \mathrm{m}$. We use the observations made by the FIS instrument in the wide far-infrared bands of $90 \mu \mathrm{m}$ and $140 \mu \mathrm{m}$. The accuracy of the calibration is currently estimated to be $26 \%$ at $90 \mu \mathrm{m}$ and $33 \%$ at $140 \mu \mathrm{m}$, and the beam sizes of these two bands are $\sim 39^{\prime \prime}$ and $58^{\prime \prime}$, respectively. For details of the AKARI far-infrared all sky survey, see Doi et al. (2009)

\subsection{Molecular line data}

We have carried out "fast" observations of different $\mathrm{CO}$ isotopic lines in some 60 Planck cold core candidate fields for the Herschel follow-up programme, five of which are included in the present sample.

The Onsala $20-\mathrm{m}$ telescope was used for ${ }^{12} \mathrm{CO} J=1 \rightarrow 0$, ${ }^{13} \mathrm{CO} J=1 \rightarrow 0$, and $\mathrm{C}^{18} \mathrm{CO} J=1 \rightarrow 0$ observations in December 2009 and April 2010. An area of a few arcmin in diameter was mapped around the position of the Planck source S3 and the sources in the SDP fields S8 and S9. The observations were carried out in frequency switching mode, with frequency throws of $\pm 10 \mathrm{MHz}$. The Onsala beam size is approximately $\sim 33^{\prime \prime}$ and the typical rms noise in the ${ }^{13} \mathrm{CO}$ and $\mathrm{C}^{18} \mathrm{O}$ spectra was below $0.1 \mathrm{~K}$ per a channel of $0.07 \mathrm{~km} \mathrm{~s}^{-1}$.

The APEX observations of field S10 were made in July 2010. The size of the ${ }^{13} \mathrm{CO} J=2 \rightarrow 1$ map is $\sim 5^{\prime}$ and the typical rms noise is below $0.2 \mathrm{~K}$. The observations were made in position switching mode, using a fixed off position of $\alpha=12^{\mathrm{h}} 18^{\mathrm{m}} 58.19^{\mathrm{s}}$ and $\delta=-71^{\mathrm{d}} 42^{\prime} 30.81^{\prime \prime}(J 2000)$. The APEX beam size at $220 \mathrm{GHz}$ is $\sim 28^{\prime \prime}$.

The IRAM-30m observations were performed in July and October 2010. The EMIR receivers E090 (3 mm) and E230 $(1 \mathrm{~mm})$ were used in parallel with the high resolution correlator
(VESPA). The most important parameters for the different settings are given in the Appendix in Table A.1. Maps of $3^{\prime} \times 3^{\prime}$ were performed using on-the-fly (OTF) mode combined with frequency switching. A summary of the observations and the main results obtained are presented in Sect. 4.3.3.

\section{Methods}

\subsection{Photometry and SEDs}

\subsubsection{Planck and IRAS photometry}

The method for estimating the photometry for the C3PO catalogue has been described in detail in Montier et al. (2010) and Paper I (Sect. 2.4). We recall here only the main steps of the detection and flux extraction process:

1. for each pixel, and for each frequency, the warm background colour $\left(C_{\mathrm{bkg}}\right)$ is estimated as the median value of the ratio of the Planck to $100 \mu \mathrm{m}$ emission maps $\left(I_{v} / I_{100}\right)$ within a $15^{\prime}$ radius disc;

2. for each Planck frequency, the contribution of the warm component is obtained by extrapolation from $100 \mu \mathrm{m}$ through $I_{v}^{\mathrm{w}}=C_{\mathrm{bkg}} \times I_{100}$;

3 . the cold residual map is computed by subtracting the warm component from the Planck map;

4. the cold source detection is performed using a thresholding method applied on the cold residual map, with the criterion $S / N>4$

5. the source shape is estimated by fitting a $2 \mathrm{D}$ elliptical Gaussian to the colour map $I_{857} / I_{100}$;

6. the flux density at $100 \mu \mathrm{m}$ is derived by fitting an elliptical Gaussian plus a polynomial surface for the background;

7. the warm template at $100 \mu \mathrm{m}$ is corrected by removing the source contribution that was estimated in the previous step;

8. aperture photometry at 857,545 , and $353 \mathrm{GHz}$ is performed on the cold residual maps, with the aperture determined by the source shape from step 5 .

The photometric uncertainties associated with this method have been estimated with a Monte Carlo analysis (see Paper I, Sect. 2.5); they are $40 \%$ for IRAS $100 \mu \mathrm{m}$ and $8 \%$ in the Planck bands. The additional calibration uncertainties to be taken into account are $13.5 \%$ and $7 \%$, respectively, for IRIS (Miville-Deschênes \& Lagache 2005) and the HFI bands 857, 545, and $353 \mathrm{GHz}$ (Planck HFI Core Team 2011b). As described 
in Paper I, the elliptical Gaussian fit performed in step 5 leads to an estimate of the minor and major axis lengths ( $\sigma_{\text {Min }}$ and $\sigma_{\text {Maj }}$, respectively), related to the FWHM values, i.e., $\theta_{\text {Min }}$ and $\theta_{\text {Maj }}$ of a Gaussian by $\sigma=\theta / \sqrt{8 \ln (2)}$. This method does not include any beam deconvolution. The fluxes and FWHM values are given for each source in Table 1, and the corresponding elliptical fit contours (traced with $\theta_{\text {Min }}$ and $\theta_{\text {Maj }}$ as minor and major axis lengths) are shown in Figs. 3-5.

The $353 \mathrm{GHz}$ band includes some contribution from the $\mathrm{CO}$ $J=3 \rightarrow 2$ molecular line. The magnitude of this effect has been estimated in Planck HFI Core Team (2011b) using data from ${ }^{12} \mathrm{CO} J=1 \rightarrow 0$ surveys, together with an estimated average line ratio of $J=3 \rightarrow 2$ to $J=1 \rightarrow 0$, and knowledge of the $353 \mathrm{GHz}$ band spectral response. The derived correction factor is $171 \mu \mathrm{K}$ in thermodynamic temperature for a CO $J=1 \rightarrow 0$ line area of $1 \mathrm{~K} \mathrm{~km} \mathrm{~s}^{-1}$. The correction should be small, but the exact effect is hard to estimate for our sources because of the lack of high spatial resolution $\mathrm{CO}$ data and because the $\mathrm{CO}$ excitation towards cold cores may differ significantly from the average values assumed in deriving the above factor. We therefore present results without making a correction for the $\mathrm{CO}$ contribution, but comment on its possible influence later.

\subsubsection{Photometry with ancillary data}

When using Herschel and AKARI data, the fluxes are estimated at a different resolution and with direct aperture photometry. The maps are convolved to a resolution of $37^{\prime \prime}$ (for Herschel and AKARI $90 \mu \mathrm{m}$ ), 58" (for Herschel and AKARI $90 \mu \mathrm{m}$ and $140 \mu \mathrm{m}$ ), or $4.5^{\prime}$ (when including Planck and IRIS data). In each case the aperture has a radius equal to the FWHM of the smoothed data and the background is subtracted using the $30 \%$ quantile value within a reference annulus that extends from $1.3 \times$ FWHM to $1.8 \times$ FWHM. In order not to be affected by possible emission from transiently heated grains, the fits employ only data at wavelengths longer than $100 \mu \mathrm{m}$. The statistical uncertainty of the flux values is derived from the surface brightness fluctuations in the reference annulus. As above, the calibration uncertainty is taken to be $7 \%$ for the Planck channels and $13.5 \%$ for IRIS $100 \mu \mathrm{m}$. For Herschel we assume a $15 \%$ calibration uncertainty for PACS and $12 \%$ for SPIRE.

\subsection{Estimation of temperatures, spectral indices, and optical depths}

Since the dust thermal emission is optically thin in the submillimetre range, the source SEDs can be modelled as a modified blackbody of the form

$S_{v}=\tau_{v} B_{v}\left(T_{\mathrm{c}}\right) \Omega_{\mathrm{cl}}$

where $S_{v}$ is the flux density integrated over the clump solid angle $\Omega_{\mathrm{cl}}$ (common to all frequencies), $\tau_{v}=N_{\mathrm{H}_{2}} \mu m_{\mathrm{H}} \kappa_{v_{0}}\left(v / v_{0}\right)^{\beta}$ is the dust optical depth, and $B_{v}$ is the Planck function at the dust colour temperature, $T_{\mathrm{c}}$. Here $N_{\mathrm{H}_{2}}$ is the $\mathrm{H}_{2}$ gas column density, $\mu=2.33$ the mean mass per particle, $m_{\mathrm{H}}$ the mass of the proton, $\kappa_{v_{0}}$ the mass absorption coefficient at frequency $\nu_{0}$ and $\beta$ the dust emissivity spectral index. The SED may be rewritten as

$S_{v}=A B_{v}\left(T_{\mathrm{c}}\right) v^{\beta}$

to separate the three quantities (assumed independent) which can be derived from the fit: amplitude $A \propto N_{\mathrm{H}_{2}} \kappa_{v_{0}}$; spectral index $\beta$; and effective temperature $T_{\mathrm{c}}$. The $\chi^{2}$ minimisation search operates in the $\left(A, T_{\mathrm{c}}, \beta\right)$ space and takes into account the colour corrections for the Planck bands described in Planck HFI Core Team (2011a). In the fitting procedure, we consider only the flux error bars associated with the photometry method, i.e., $40 \%$ for IRIS and $8 \%$ for HFI bands. In a second step, we compute the final error bars on $T_{\mathrm{c}}$ and $\beta$ by adding the contribution from calibration uncertainties; these have to be considered separately because the HFI calibration errors are not independent of eachother. In Paper I the size of this effect was estimated using a grid of $T_{\mathrm{c}}$ and $\beta$ values, and was found to be small $\left(\leq 2.5 \%\right.$ for $T_{\mathrm{c}}$ and $\leq 2 \%$ for $\beta$ ) compared to the uncertainties of the flux extraction method. The final uncertainties in $T_{\mathrm{c}}$ and $\beta$ are obtained as a quadratic sum of the two contributions. They do not include the correlations between $T_{\mathrm{c}}$ and $\beta$ inherent in the fitting procedure itself.

Applying the same fitting method pixel by pixel to the IRIS $100 \mu \mathrm{m}$ and HFI 857, 545, and $353 \mathrm{GHz}$ surface brightness images $\left(1^{\circ} \times 1^{\circ}\right)$, we calculate maps of the dust colour temperature and the emissivity index. Here the flux error bars are the quadratic sum of the noise map and the calibration uncertainties. The averaged column densities of the clumps are then derived from the observed flux at $v_{0}=857 \mathrm{GHz}$ and the dust colour temperature inferred from the fit:

$N_{\mathrm{H}_{2}}=\frac{S_{v_{0}}}{\Omega_{\mathrm{cl}} \mu m_{\mathrm{H}} \kappa_{v_{0}} \times B_{v_{0}}\left(T_{\mathrm{c}}\right)}$

with $\Omega_{\mathrm{cl}}=\pi \sigma_{\mathrm{Maj}} \sigma_{\mathrm{Min}}$.

The value of $\kappa_{v}$ is a main source of uncertainty. Large variations exist between dust models, depending on properties of the dust (see Beckwith et al. 1990; Henning et al. 1995): composition (with or without ice mantles); structure (compact or fluffy aggregates); and size. Both models and observations show that $\kappa_{v}$ increases from the diffuse medium to dense and cold regions by a factor of 3-4 (Ossenkopf \& Henning 1994; Kruegel \& Siebenmorgen 1994; Stepnik et al. 2003; Juvela et al. 2011; Planck Collaboration 2011u). For this study, we adopt the dust absorption coefficient of Beckwith et al. (1990) inferred for highdensity environments (Preibisch et al. 1993; Henning et al. 1995; Motte et al. 1998):

$\kappa_{v}=0.1 \mathrm{~cm}^{2} \mathrm{~g}^{-1}(v / 1000 \mathrm{GHz})^{\beta}$,

with a fixed emissivity index of $\beta=2$. The reference frequency $v_{0}=857 \mathrm{GHz}$ chosen to estimate $N_{\mathrm{H}_{2}}$ is such that the extrapolation from $1000 \mathrm{GHz}$ with $\beta=2$ (instead of the fitted spectral index) introduces an uncertainty on $N_{\mathrm{H}_{2}}$ of less than $30 \%$. At a distance $d$, the mass of the clump is simply $M=N_{\mathrm{H}_{2}}^{\mathrm{cl}} \Omega_{\mathrm{cl}} \mu m_{\mathrm{H}} d^{2}$ or

$$
M=\frac{S_{v_{0}} d^{2}}{\kappa_{v_{0}} B_{v_{0}}\left(T_{\mathrm{c}}\right)} .
$$

\section{Results}

\subsection{Physical parameters derived from Planck and IRIS data}

The cold clump SEDs determined using the Planck HFI and IRIS flux densities are shown in Fig. 2. As discussed in Sect. 3.1.1, these SEDs are those of the cold dust residual. The values obtained for the dust temperature and emissivity spectral index are shown in the frames of the figure. Using the method described in Sect. 3.2, we infer the gas column density, bolometric luminosity, and mass of each clump (Table 2). All these parameters $\left(T, \beta, N_{\mathrm{H}_{2}}\right)$ are values which are averaged over the 

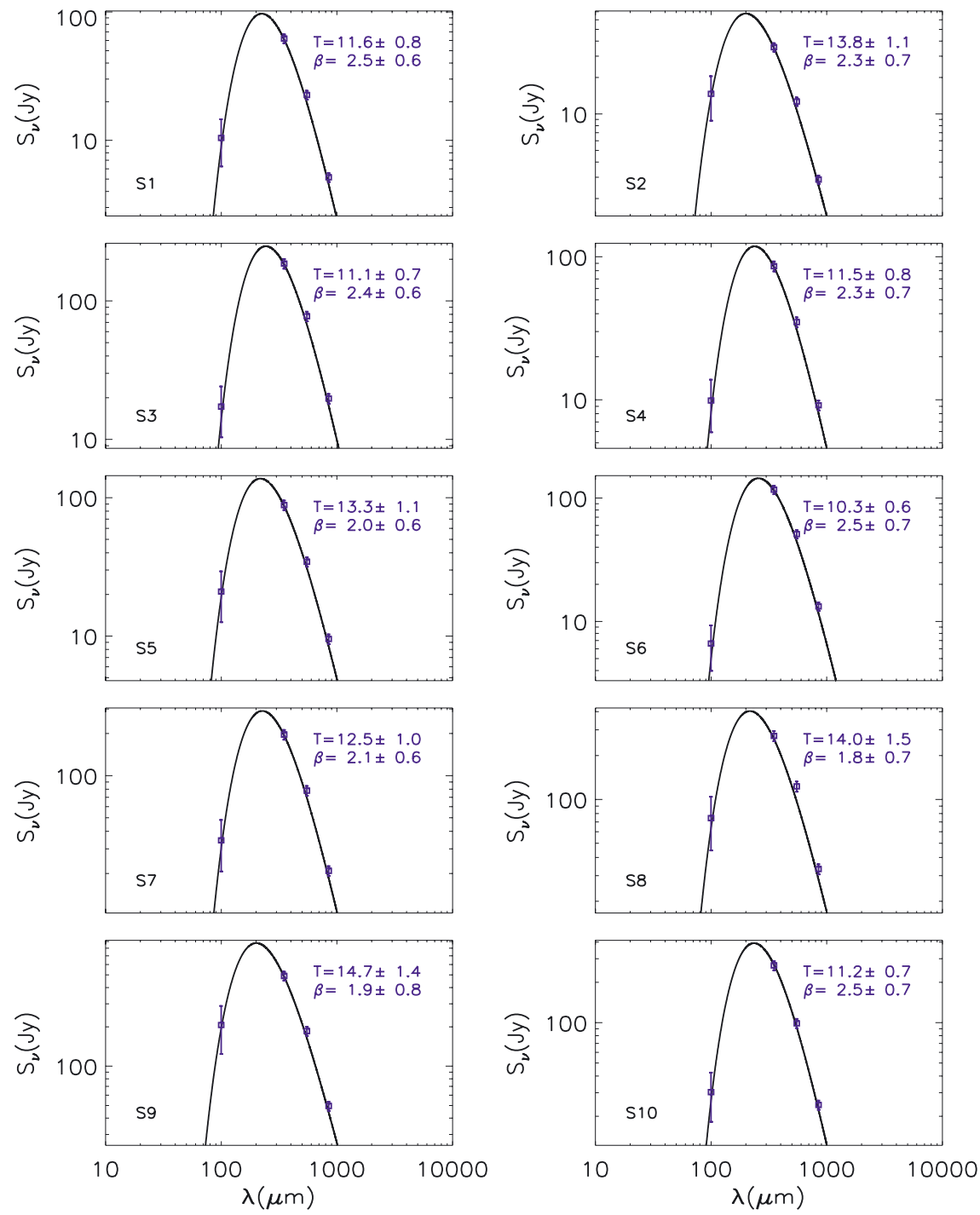

Fig. 2. SEDs and fit parameters $(T, \beta)$ obtained combining Planck HFI and IRIS flux densities integrated over the clump in the cold residual maps. line-of-sight and the extent of the cold clumps, which reach the parsec-scale. Denser and/or colder structure is to be expected on smaller scales. These averaged temperatures vary between 10.3 and $14.7 \mathrm{~K}$, and the dust emissivity spectral indices range between 1.8 and 2.5 .

The column densities are distributed around a mean of $7.5 \times 10^{21} \mathrm{~cm}^{-2}$, with a few of the largest values being above $\sim 10^{22} \mathrm{~cm}^{-2}$. These relatively moderate column densities also correspond to averaged values over the extent of the clumps. The clumps are likely to be heterogeneous, with denser substructures that will be studied with higher angular resolution data in Sect. 4.3.1. The masses of the sample cover a large range from 3.5 to $1800 M_{\odot}$. The highest-mass object, S6, is also the coldest source of the sample, and it is quite extended $(\sim 2.9 \times 6.7 \mathrm{pc})$; these properties make this source an interesting candidate for being a high mass star-forming precursor in its early stages. More generally, the sizes of the source sample are rather large, in the range $0.2-11$ parsecs, which confirms that they should be better classified as "clumps" rather than "cores," according to the terminology used for nearby molecular clouds (e.g., Williams et al. 2000; Motte et al. 2007). Scales typically considered for "dense cores" and "clumps" are $\sim 0.1 \mathrm{pc}$ and $\sim 1 \mathrm{pc}$, respectively.

As noted in Paper I, most of the cold clumps found with the CoCoCoDet procedure are elongated, with aspect ratios significantly larger than unity. The maps of Fig. 1 show that these clumps are not isolated, but are, in many cases, substructures of long filaments, not always straight. Without direct estimates of the density it is difficult to assess that they are actual filaments, denser than their environment. However, their brightness contrast above the background is a few, in the case of S4, S5, S6 and S10, suggesting actual density enhancements given their small thickness. Their length, estimated from their angular size in the maps of Fig. 1 and their distance, ranges from about $5 \mathrm{pc}$ for $\mathrm{S} 10$ to $18 \mathrm{pc}$ for $\mathrm{S} 5$.

Because of this, we choose to focus not on the clump mass, that depends on the size of the clump derived from the detection procedure, but on the mass per unit length of the filament at the position of the cold clump. This mass per unit length (or linear mass density) is given by $m=16.7 M_{\odot} \mathrm{pc}^{-1} N_{21} \theta_{\text {Min }}$, where $N_{21}$ is the clump column density expressed in units of $10^{21} \mathrm{~cm}^{-2}$ and $\theta_{\text {Min }}$, the half-power thickness of the clump identified in the Planck maps, is taken as the width of the filament. The linear mass densities are given in Table 2 for all clumps, including those with an aspect ratio only slightly larger than unity. However, they should be considered only as an approximate guide to the linear mass density at the location of the clump.

The linear mass densities vary by a factor $\sim 30$ and compare well with those inferred from molecular lines. The range of values $\left(15\right.$ to $\left.400 M_{\odot} \mathrm{pc}^{-1}\right)$ is characteristic of regions of massive or intermediate-mass star formation, such as Orion and $\rho$ Oph 

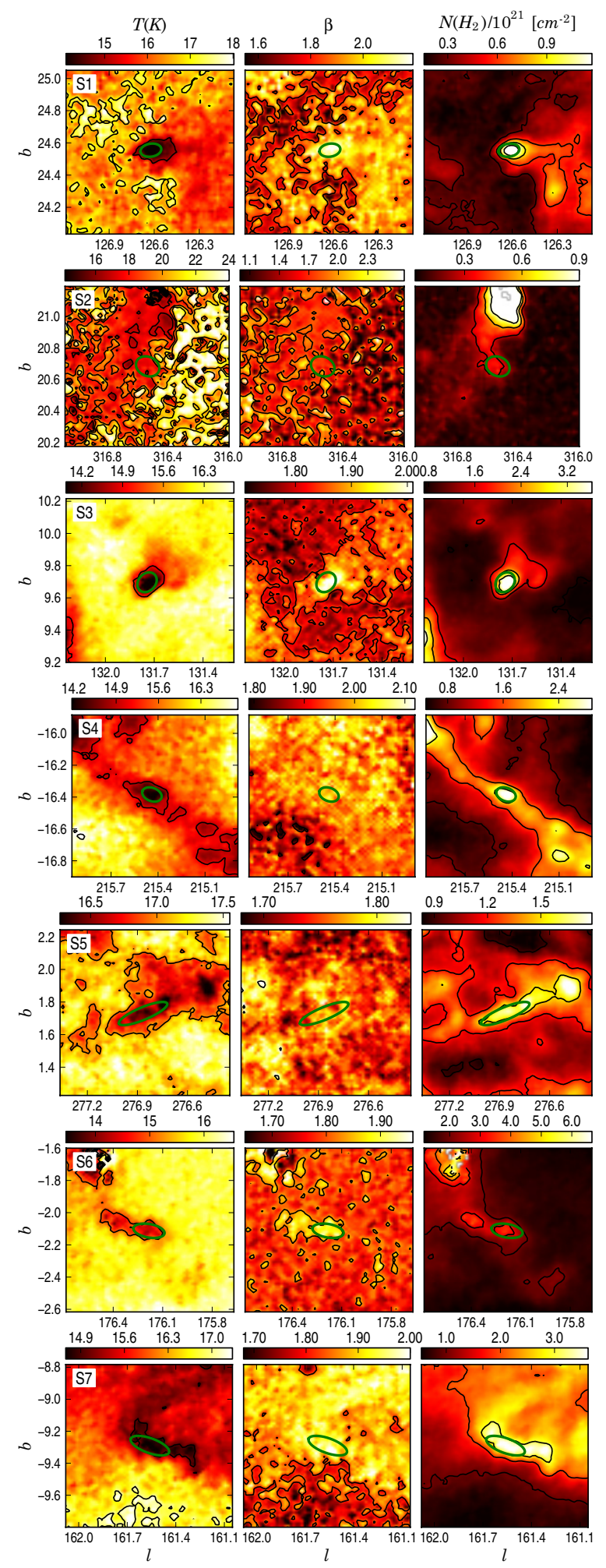

Fig. 3. Maps of dust colour temperature, emissivity spectral index and column density for each clump field (the three SDP fields are shown in Juvela et al. (2011) using Herschel maps). The $N_{\mathrm{H}_{2}}$ contours correspond to the ticks on the colour bar. For $\mathrm{T}$ and $\beta$, the contours are shown respectively at $2 \mathrm{~K}$ intervals and for $\beta=1.85$. The ellipses trace the FWHMs of the Planck clumps as inferred from the fit (see Table 1).

(Hily-Blant et al. 2004). The linear mass densities of the most tenuous filaments $\left(<1 M_{\odot} \mathrm{pc}^{-1}\right.$, Falgarone et al. 2001) are not found in the present sub-sample of Planck cold clumps.
Many of the cold clumps detected by Planck therefore appear as cold substructures within larger scale filamentary structures that have length up to $\sim 20 \mathrm{pc}$ in the Planck maps and with parsec-scale thickness (S5, S6, S7). The thinnest filaments found are only a few parsec long and with thickness of a few $0.1 \mathrm{pc}$ (S10).

At this stage of the analysis, it is difficult to assess the exact nature of the sources, i.e., discriminating between protostellar objects and starless clumps. We can however use the temperature and bolometric luminosity estimates as first indicators of the evolutionary stage of the sources. Such an approach was proposed by Netterfield et al. (2009) and Roy et al. (2011) in their analysis of the cold cores detected with the balloon-borne experiment, BLAST, in the Cygnus-X and Vela surveys. Among the values obtained for the ratio $L / M$ in our sample (ranging from 0.13 to $0.91 L_{\odot} / M_{\odot}$ ), it is interesting to note that the three highest (with $L / M>0.6 L_{\odot} / M_{\odot}$ ) are associated with $T>14 \mathrm{~K}$ dust sources S2, S8 and S9). As we will see in Sect. 4.3.1, the Planck clumps in these fields harbour warm dust sources, and two of them are associated with young stellar objects.

\subsection{Colour temperature and spectral index maps}

Figure 3 shows the $1^{\circ} \times 1^{\circ}$ maps for dust colour temperature and dust spectral index that were estimated using the IRAS $100 \mu \mathrm{m}$ data and the three highest frequency HFI channels (see Sect. 3.2) without background removal. The three Herschel SDP fields (S8, S9 and S10) are not presented here, because the analysis of $T$ and $\beta$ was already described in detail in Juvela et al. (2011).

Except for the faint (and not so cold) source S2, a clear signature of cold dust emission is visible directly on these temperature maps at the location of the clumps. The colour temperature characterizing the line-of-sight integrated intensity in the direction of the clump is typically about $14 \mathrm{~K}$, warmer by a few Kelvin than the clump temperature deduced from its SED after warm background subtraction. The clumps are embedded within extended structures in the temperature range $16-18 \mathrm{~K}$. For reference, dust in the diffuse medium of the local Galactic neighbourhood has a temperature of around 17.5 K (Boulanger et al. 1996; Schlegel et al. 1998). In several cases (S4, S5, S6 and S7) the cold clumps appear as the coldest spot (or one of the coldest spots) along filamentary structures, which are already colder than the larger scale environment. The temperature and column density maps are correlated, with the coldest structures corresponding, as expected, to the most opaque parts of the field at the angular resolution probed by Planck. The sharp edges seen in the intensity maps of the fields S1, S3 and S7 are also clearly associated with column density sharp transition. This may be the signature of shock compression and triggering core formation that should be investigated in further studies.

We have compared the results on column densities with extinction measurements. Extinction is calculated with the NICER method (Lombardi \& Alves 2001) using stars from the 2MASS catalogue (Skrutskie et al. 2006). The $A_{V}$ maps of the fields are derived assuming an extinction curve that corresponds to $R_{V}=3.1$. Preliminary extinction maps are created using nearby fields where IRAS $100 \mu \mathrm{m}$ data indicates a low dust column density. The $A_{V}$ maps obtained with this method are shown in the Appendix (in Fig. A.6) at $4.5^{\prime}$ resolution. A clear signature is visible, with an increase by about a factor of 2 in $A_{V}$ toward the clumps. The $A_{V}$ values derived ( 2-5 mag) are consistent with the previous $N_{\mathrm{H}_{2}}$ estimates of several times $10^{21} \mathrm{~cm}^{-2}$, derived from the SEDs. 
Planck Collaboration: Planck early results. XXII.
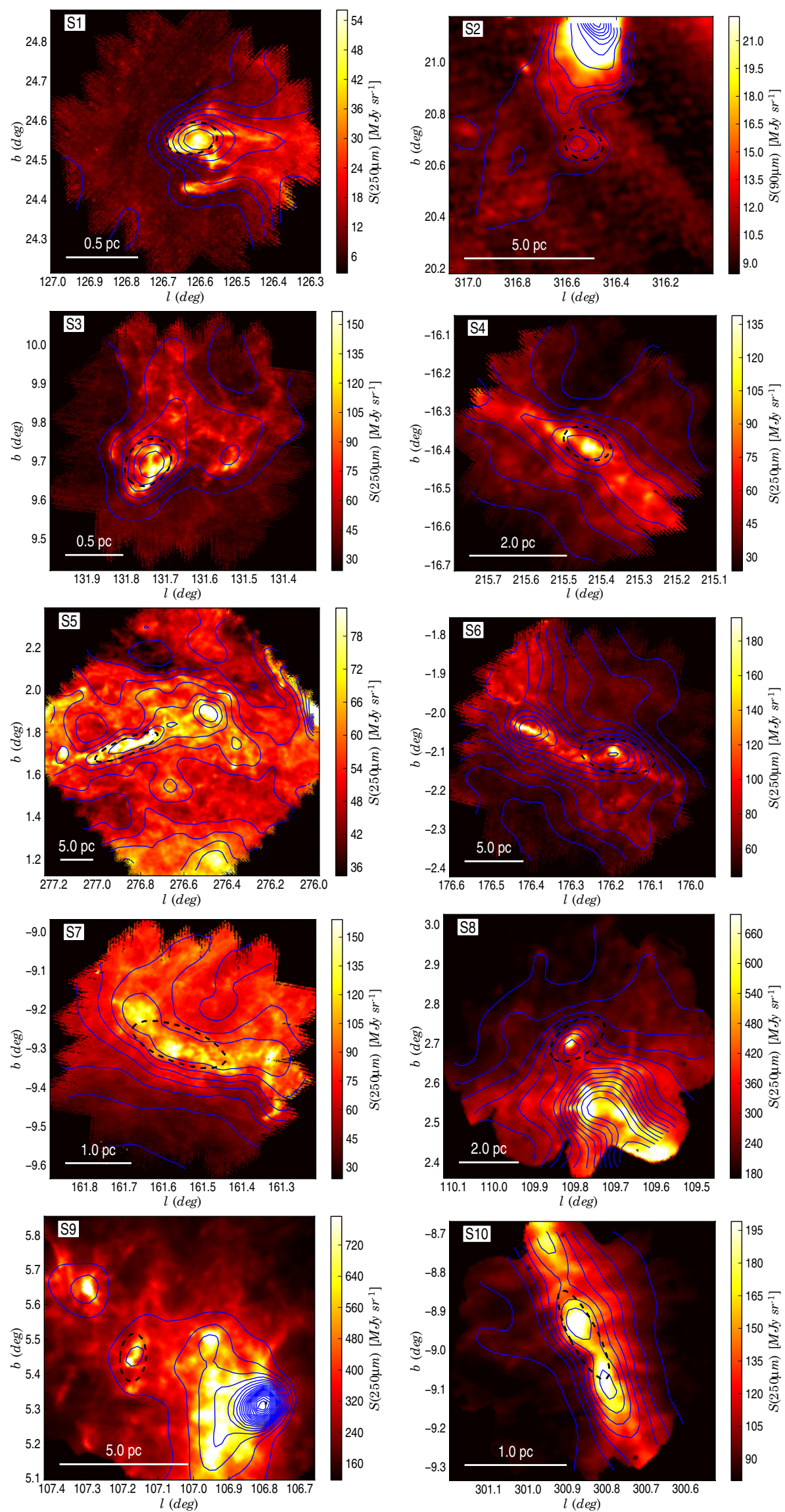

Fig. 4. Planck $857 \mathrm{GHz}$ surface brightness contours on Herschel SPIRE maps at $250 \mu \mathrm{m}$. The source $\mathrm{S} 2$ has not been observed with Herschel and the displayed image corresponds to the AKARI $90 \mu \mathrm{m}$ wide filter. As in Fig. 3, the dashed ellipses are tracing the estimated size of the Planck cold clump. 
Table 2. Clump physical parameters derived from Planck SEDs.

\begin{tabular}{lccccccrrc}
\hline \hline Source & $\begin{array}{c}T_{\mathrm{c}} \\
(\mathrm{K})\end{array}$ & $\beta$ & $\begin{array}{c}\theta_{\mathrm{Min}}{ }^{a} \\
(\mathrm{pc})\end{array}$ & $\begin{array}{c}\theta_{\mathrm{Maj}}{ }^{a} \\
(\mathrm{pc})\end{array}$ & $\begin{array}{c}N_{\mathrm{H}_{2}} \\
\left(\mathrm{~cm}^{-2}\right)\end{array}$ & $\begin{array}{c}m^{b} \\
\left(M_{\odot} \mathrm{pc}^{-1}\right)\end{array}$ & $\begin{array}{c}M \\
\left(M_{\odot}\right)\end{array}$ & $\begin{array}{c}L \\
\left(L_{\odot}\right)\end{array}$ & $\begin{array}{c}L / M \\
\left(L_{\odot} / M_{\odot}\right)\end{array}$ \\
\hline S1 & $11.6 \pm 0.8$ & $2.5 \pm 0.6$ & 0.2 & 0.3 & $4.4 \times 10^{21}$ & 15 & 3.5 & 1.0 & 0.28 \\
S2 & $13.8 \pm 1.1$ & $2.3 \pm 0.7$ & 1.2 & 1.5 & $8.0 \times 10^{20}$ & 16 & 15.0 & 11.0 & 0.72 \\
S3 & $11.1 \pm 0.7$ & $2.4 \pm 0.6$ & 0.4 & 0.5 & $1.1 \times 10^{22}$ & 77 & 22.0 & 4.3 & 0.19 \\
S4 & $11.5 \pm 0.8$ & $2.3 \pm 0.7$ & 0.7 & 1.0 & $6.3 \times 10^{21}$ & 74 & 44.0 & 11.0 & 0.24 \\
S5 & $13.3 \pm 1.1$ & $2.0 \pm 0.6$ & 2.7 & 11.0 & $1.7 \times 10^{21}$ & 77 & 520.0 & 270.0 & 0.52 \\
S6 & $10.3 \pm 0.6$ & $2.5 \pm 0.7$ & 2.9 & 6.7 & $8.4 \times 10^{21}$ & 400 & 1800.0 & 230.0 & 0.13 \\
S7 & $12.5 \pm 1.0$ & $2.1 \pm 0.6$ & 0.6 & 1.5 & $4.9 \times 10^{21}$ & 49 & 45.0 & 17.0 & 0.37 \\
S8 & $14.0 \pm 1.5$ & $1.8 \pm 0.7$ & 1.2 & 2.2 & $8.1 \times 10^{21}$ & 160 & 210.0 & 140.0 & 0.65 \\
S9 & $14.7 \pm 1.4$ & $1.9 \pm 0.8$ & 1.0 & 1.9 & $1.6 \times 10^{22}$ & 270 & 330.0 & 300.0 & 0.91 \\
S10 & $11.2 \pm 0.7$ & $2.5 \pm 0.7$ & 0.3 & 1.0 & $1.3 \times 10^{22}$ & 64 & 40.0 & 8.4 & 0.21 \\
\hline
\end{tabular}

Notes. ${ }^{(a)}$ Minor and major axis FWHM values of the Gaussian ellipse, converted to length units. ${ }^{(b)}$ Linear mass density.

In most fields the colour temperature and the spectral index are seen to be anticorrelated, with high spectral indices being found at the location of the temperature minima. The parameters $T$ and $\beta$ are known to be connected so that any noise present in the observations tends to produce a similar anticorrelation. However, the spatial coherence of the $T$ and $\beta$ maps strongly suggests that the results of Fig. 3 are not caused by statistical noise. There is still the possibility of systematic errors, but the similarity of the results obtained in separate fields makes this unlikely. A pure calibration error would change the $\beta$ and $T$ values in a systematic way but would not significantly affect the dispersion of the spectral index values (Juvela et al. 2011). The line-ofsight temperature variations tend to decrease the observed spectral indices. The effect should be stronger toward the cold clump. The fact that $\beta$ at those positions is larger than in the diffuse areas suggests that the emissivity index of the grains has increased in the cores more than what is visible in the maps, i.e than that inferred from the line-of-sight integrated SEDs (see Malinen et al. 2011). Therefore, the results support the idea of spatial variation of grain properties that could result from temperature-dependent processes in the dust emission mechanism (Meny et al. 2007; Boudet et al. 2005; Coupeaud et al. 2011, see Paper I discussion). We have estimated the effect of the correction of the $\mathrm{CO}$ contribution to the $353 \mathrm{GHz}$ fluxes using the $\mathrm{CO}$ data survey from Dame et al. (2001), which covers all sources except S2. This has been performed on the SEDs obtained using the large aperture, which is closer to the angular resolution of the $\mathrm{CO}$ data. The results lead to a very small change of the fit parameter values, typically with a $\sim 3 \%$ increase of $\beta$, and a decrease of the colour temperatures by a few $0.1 \mathrm{~K}$.

\subsection{Analysis using ancillary data}

\subsubsection{Herschel and AKARI maps}

For all sources we have higher resolution dust continuum data in the form of Herschel (resolution 37" or better) and/or AKARI ( $58^{\prime \prime}$ or better) maps. These are used: to examine internal structure of the sources; to derive physical parameters of the Planck sources and compare them with the results of Sect. 4.1; and to study the properties of the structures found at scales below the Planck beam size.

Figure 4 shows Herschel $250 \mu$ m data (resolution $\sim 18^{\prime \prime}$ ) for all, but one, sources. S2 has not been observed with Herschel and we show the AKARI $90 \mu \mathrm{m}$ map instead $\left(\sim 39^{\prime \prime}\right)$. For comparison, the Planck $857 \mathrm{GHz}$ brightness contours are overplotted and ellipses show the cold clump sizes as derived from elliptical Gaussian fits on the cold residual maps (cf. Paper I and Sect. 3.1.1). A blow-up of the Herschel $250 \mu \mathrm{m}$ maps is shown in Fig. 5.

In most fields, a significant amount of structure at scales below the Planck resolution is visible. The Musca field (S10) is an exception. The Herschel observations there resolve the filament but do not reveal further structure within the Planck-detected clump. Interestingly, this field is among the closest to the Sun. The other filamentary structures (S4, S5, S6, and S7) break up into numerous smaller bright knots, the brightest often coinciding with the position of the Planck detection. The two cometary shaped clouds (S1 and S3) similarly harbour a number of bright smaller knots and narrow filaments that show up with a large contrast $(>2)$ above an extended background.

\subsubsection{Photometry with Planck, Herschel and AKARI}

We have performed fits on the SEDs of selected substructures within the Planck cold clumps to further characterise their inner structure. The aperture photometry is performed after subtraction of a background level estimated as described in Sect. 3. The radius of the largest aperture is $4.5^{\prime}$ (i.e., a diameter of 540"). For the substructures, we use apertures of either $74^{\prime \prime}$ or $116^{\prime \prime}$ diameter. The locations of these apertures are drawn in Fig. 5 and listed in Table 3. The SED fits involve data at wavelengths longer than $100 \mu \mathrm{m}$. This removes the problem of a possible contribution from transiently heated grains to the $100 \mu \mathrm{m}$ flux densities, but also reduces the effects of the line-of-sight temperature variations.

Figure 6 illustrates the various SED estimates for the S1 and S5 fields. It displays the Planck SED as given in the C3PO catalogue (therefore corrected for the warm background contribution) and the aperture photometry using smaller apertures on the brightest substructure of the S1 field (Fig. 5). For the large apertures, the flux densities from Planck and Herschel are in good agreement with each other; in fact it appears that the assumed sources of uncertainty may overestimate the errors. The temperatures obtained for the fixed apertures (Table 3) are the same within the error bars as those derived from the Planck and IRIS data only (Table 2). It is therefore most encouraging that the estimates based only on Planck and IRIS data do not significantly change when the five Herschel bands are added that better cover the short wavelength side of the SED maxima. The modified blackbody parameters derived from the fits are listed in Table 3. 
Planck Collaboration: Planck early results. XXII.
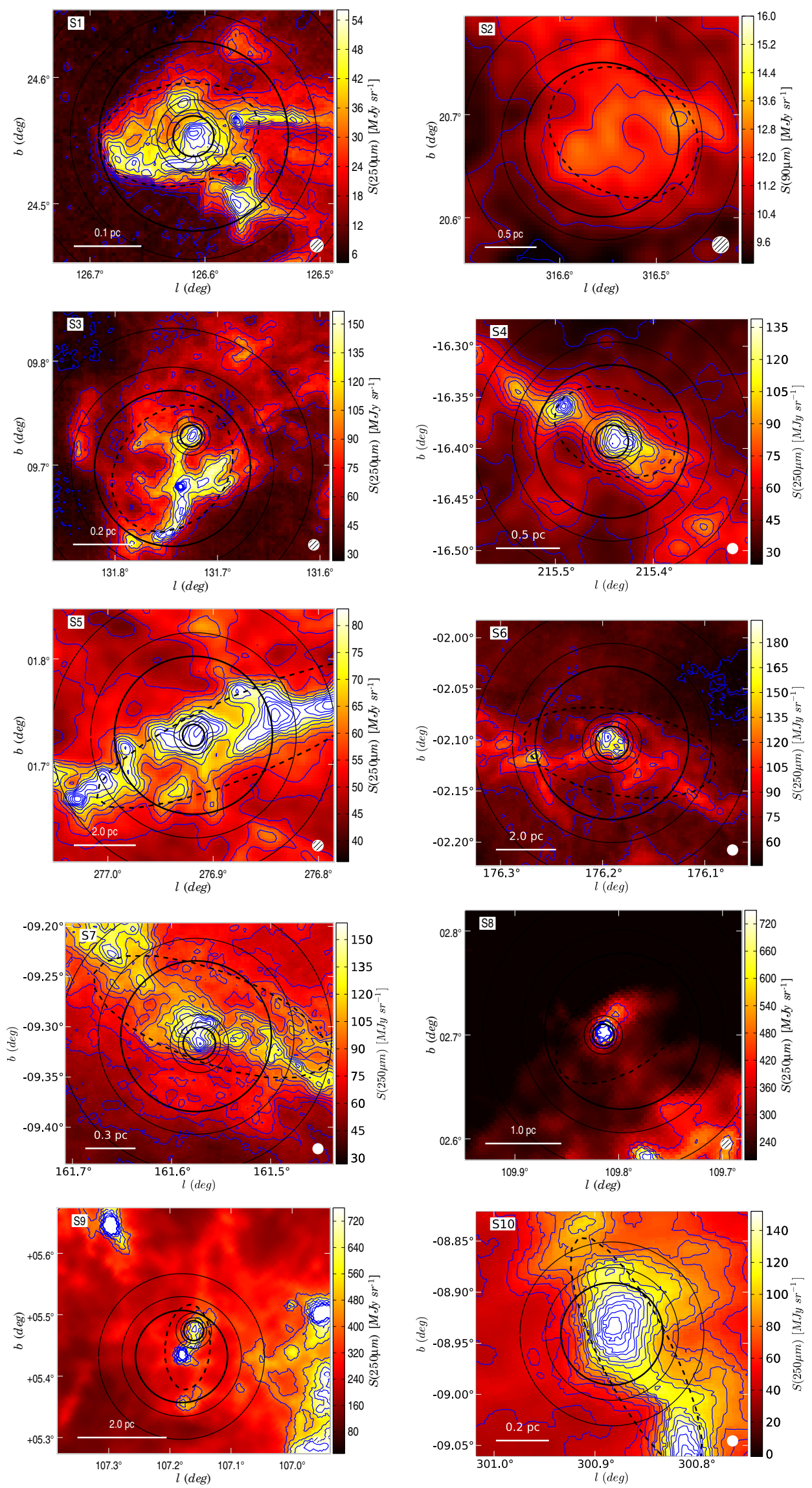

Fig. 5. Blow-up of Herschel SPIRE $250 \mu \mathrm{m}$ maps at the location of the Planck cold clumps localised by their elliptic boundary. The different circles and the annuli refer to the apertures adopted for the photometry used in the SEDs. The large aperture has a $9^{\prime}$ diameter, and the smaller ones correspond to either 74" (S3 and S5), 116" (S1, S4, S6, S7, S8 and S9), or 360" (S10). 

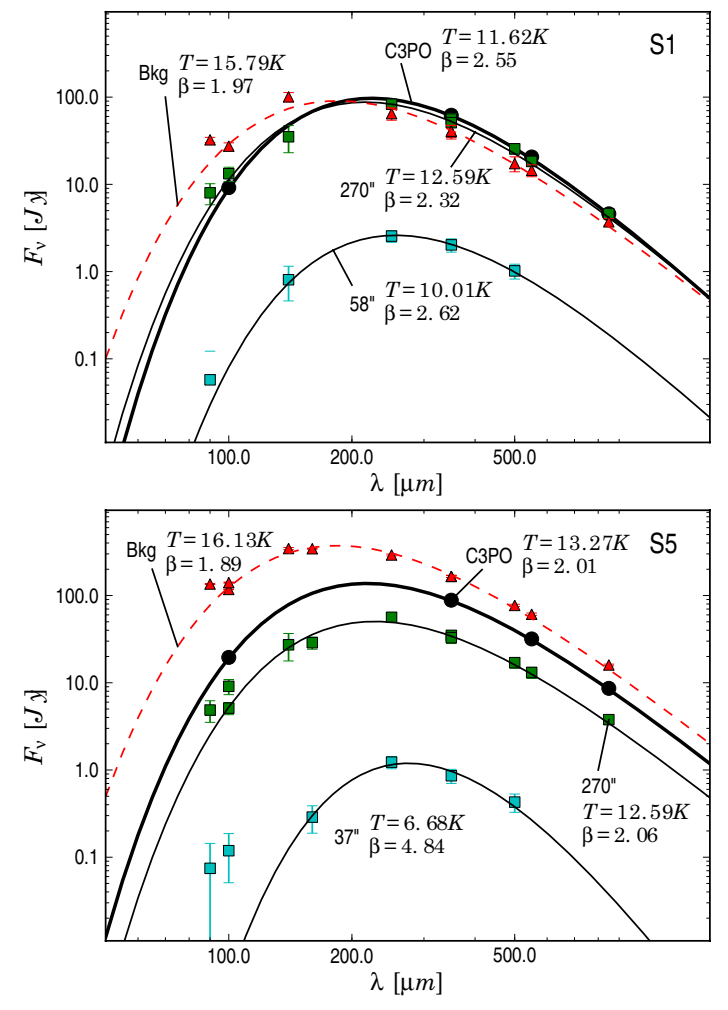

Fig. 6. Spectral energy distribution of various components in the S1 (upper panel) and S5 (lower panel) fields, including: the Planck SED according to the $\mathrm{C} 3 \mathrm{PO}$ catalogue (solid circles and thick solid line), aperture photometry with $270^{\prime \prime}$ and $37^{\prime \prime}$ or $58^{\prime \prime}$ radius aperture sizes (squares and thin solid lines) and the background SED (triangles and dashed line). The temperatures and $\beta$ values derived from the modified blackbody fits are given in the figure (see also Table 3 ). The background SED is that of the median surface brightness in the reference annulus surrounding the larger aperture (see Fig. 5).

One of the most interesting results is the low temperatures obtained for the SEDs in the 74" apertures. Except for sources S8 and S9, they are all significantly smaller than those obtained in the larger apertures, the lowest values being at about $7 \mathrm{~K}$. Therefore, in spite of the large telescope beam size, the sensivity of Planck is such that the cold clump detection method is actually able to pick-up and locate (within a few arcmin) structures of very cold dust that are significantly smaller than the beam.

In sources $\mathrm{S} 8$ and $\mathrm{S} 9$, the substructures are found to be warmer, with: $T \sim 19-21 \mathrm{~K}$. As presented in Juvela et al. (2010), the PACS $100 \mu \mathrm{m}$ maps have revealed the presence of bright compact sources within the Planck-detected clumps. These objects are likely to be very young stellar objects, still embedded in a more extended dense and cold cloud, whose emission is dominating the submillimetre wavelength range studied with Planck. As described in the Appendix, S8 and S9 are both located close to active star-forming regions, with $\mathrm{S} 8$ having nearby HII regions, and OB stellar associations.

We note that neither S8 or S9 has any clear counterpart in the $100 \mu \mathrm{m}$ IRAS map (see Figs. A.2 and A.3); the emission of the young stellar objects is not visible, even although this is the same wavelength as for PACS, probably because the sources are very faint, and diluted with the other components in the IRAS beam. However, for S8, both the $170 \mu \mathrm{m} I S O$ Serendipity Survey (Stickel et al. 2007) and the AKARI FIS survey at $140 \mu \mathrm{m}$ (Doi et al. 2009) showed a faint feature at this position. Shorter wavelength data with higher angular resolution would be interesting to combine for these particular sources. Among our sample, the field S8 has been observed by Spitzer, with the $24 \mu \mathrm{m}$ MIPS data revealing a number of compact sources. Within the Planck clump, the brightest submillimetre peak (PCC288-A in Juvela et al. 2010) is seen to contain at least four distinct midinfrared sources. These data will be analysed in detail in a future publication.

We have also estimated the column densities within each aperture, and the average densities and masses obtained in the smallest aperture. In most cases, the derived column densities are higher than those estimated from the Planck clump SEDs. Moreover, as we found with the linear mass densities estimated with Planck data, they vary over a factor of about 30 . These values should probably be considered as lower limits, because, as shown in Fig. 5, the annulus associated with the small apertures are close to the source and often include a fraction of signal associated with the source itself. We have estimated the impact of the background level on the derived column density by using the average brightness in the largest annulus instead of the smallest. The derived column densities are increased slightly, by up to a factor of 2 .

\subsubsection{Analysis of molecular line data}

All the Planck clumps observed in ${ }^{13} \mathrm{CO}$ and $\mathrm{C}^{18} \mathrm{O}$ lines have a clear line detection, and the small maps show peaks that coincide with the cold substructures (Figs. 7 and 8).

The ${ }^{13} \mathrm{CO}$ linewidths (Table 4 ) are typically $1-2 \mathrm{~km} \mathrm{~s}^{-1}$ and those of the $\mathrm{C}^{18} \mathrm{O}$ lines, although narrower, range between 0.3 and $1.6 \mathrm{~km} \mathrm{~s}^{-1}$. Several velocity components are present, either seen (in a few $\mathrm{C}^{18} \mathrm{O}$ spectra) as two distinct peaks, or inferred from the non-Gaussian lineshapes, as shown by S6 spectra in Fig. 7. Assuming that the dust and gas in these cold dense clumps is in thermal balance, then the gas temperature is close to $10 \mathrm{~K}$ and the sound velocity in $\mathrm{CO}$ is low, $\sim 0.05 \mathrm{~km} \mathrm{~s}^{-1}$. The molecular linewidths are therefore all suprathermal. It is interesting to compare the level of non-thermal support provided by these gas motions to the self-gravity. This can be done by comparing the gas linear mass density with its critical (or virial) value, $m_{\text {vir }}=2 \sigma^{2} / G$ which is the largest mass per unit length of a selfgravitating cylinder, given the non-thermal support provided by the internal motions of dispersion $\sigma=\Delta v / 2.35$ (Fiege \& Pudritz 2000).

The virial linear mass densities inferred from the $\mathrm{C}^{18} \mathrm{O}$ linewidths of Table 4 range between $10 M_{\odot} \mathrm{pc}^{-1}$ for $\mathrm{S} 10$, to $260 M_{\odot} \mathrm{pc}^{-1}$ for S8. A crude comparison with the linear mass densities estimated at the resolution of the Planck data (Table 2) suggests that the ratios $m / m_{\text {vir }}$ are within a factor of a few of unity. Therefore, as a first approximation, these cold clumps are in rough equilibrium between self-gravity and non-thermal support.

The column densities have been estimated from a single lineof-sight towards each object. When available (in the case of IRAM observations), we have combined the $J=1 \rightarrow 0$ and $2 \rightarrow 1$ transitions to derive the excitation temperature and the density using a Large Velocity Gradient radiative transfer model (Goldreich \& Kwan 1974). In the case of the Onsala and APEX observations, we only have data for one transition; in these cases, we assume an excitation temperature of $11 \mathrm{~K}$ and use the $\mathrm{C}^{18} \mathrm{O}$ line intensity to estimate the column density of the cores. The molecular hydrogen column densities are calculated assuming a $\mathrm{C}^{18} \mathrm{O}$ fractional abundance of $\left[\mathrm{C}^{18} \mathrm{O}\right] /\left[\mathrm{H}_{2}\right]=10^{-7}$.

The results are presented in Table 4 . The table includes estimates of the mean density, calculated assuming a core size 
Planck Collaboration: Planck early results. XXII.

Table 3. Results from SED fits based on flux densities from aperture photometry (for data at $\lambda>100 \mu \mathrm{m}$ ).

\begin{tabular}{|c|c|c|c|c|c|c|c|c|c|}
\hline Object & $\begin{array}{c}l \\
\text { (deg) }\end{array}$ & $\begin{array}{c}b \\
(\operatorname{deg})\end{array}$ & $\begin{array}{c}\text { Aperture } \\
(\operatorname{arcsec})\end{array}$ & $\begin{array}{l}\text { Aperture size } \\
\text { (pc) }\end{array}$ & $\begin{array}{c}T_{\mathrm{c}} \\
(\mathrm{K})\end{array}$ & $\beta$ & $\begin{array}{c}N_{\mathrm{H}_{2}} \\
\left(\mathrm{~cm}^{-2}\right)\end{array}$ & $\begin{array}{c}n_{\mathrm{H}_{2}} \\
\left(\mathrm{~cm}^{-3}\right)\end{array}$ & $\begin{array}{c}M \\
\left(M_{\odot}\right)\end{array}$ \\
\hline S1-1 & 126.61 & 24.55 & 540 & 0.39 & $12.59 \pm 0.61$ & $2.32 \pm 0.14$ & $9.3 \times 10^{20}$ & & \\
\hline S1-1 & 126.61 & 24.55 & 116 & 0.08 & $10.01 \pm 1.32$ & $2.62 \pm 0.54$ & $1.9 \times 10^{21}$ & & \\
\hline S1-1 & 126.61 & 24.55 & 74 & 0.05 & $9.75 \pm 1.98$ & $2.67 \pm 0.92$ & $3.0 \times 10^{21}$ & $2.7 \times 10^{4}$ & 0.13 \\
\hline S2-1 & 316.56 & 20.68 & 540 & 1.44 & $15.08 \pm 0.93$ & $1.67 \pm 0.17$ & $1.9 \times 10^{20}$ & & \\
\hline S3-2 & 131.74 & 9.70 & 540 & 0.52 & $11.26 \pm 0.35$ & $2.36 \pm 0.11$ & $3.3 \times 10^{21}$ & & \\
\hline S3-3 & 131.74 & 9.68 & 116 & 0.11 & $7.09 \pm 0.74$ & $3.96 \pm 0.78$ & $5.3 \times 10^{22}$ & & \\
\hline S3-4 & 131.73 & 9.73 & 74 & 0.07 & $8.53 \pm 4.03$ & $3.10 \pm 1.03$ & $2.0 \times 10^{22}$ & $1.4 \times 10^{5}$ & 1.54 \\
\hline S4-1 & 215.44 & -16.39 & 540 & 1.18 & $12.23 \pm 0.49$ & $2.18 \pm 0.16$ & $1.5 \times 10^{21}$ & & \\
\hline S4-1 & 215.44 & -16.39 & 116 & 0.25 & $7.02 \pm 0.34$ & $4.40 \pm 0.42$ & $3.9 \times 10^{22}$ & & \\
\hline S4-1 & 215.44 & -16.39 & 74 & 0.16 & $7.24 \pm 0.80$ & $4.02 \pm 0.45$ & $3.2 \times 10^{22}$ & $9.8 \times 10^{4}$ & 12.4 \\
\hline S5-4 & 276.92 & 1.73 & 540 & 5.24 & $12.59 \pm 0.28$ & $2.06 \pm 0.13$ & $6.1 \times 10^{20}$ & & \\
\hline S5-4 & 276.92 & 1.73 & 74 & 0.72 & $6.68 \pm 0.47$ & $4.84 \pm 0.11$ & $1.7 \times 10^{22}$ & $1.1 \times 10^{4}$ & 127.7 \\
\hline S6-2 & 176.19 & -2.10 & 540 & 5.24 & $11.50 \pm 0.37$ & $2.31 \pm 0.11$ & $1.6 \times 10^{21}$ & & \\
\hline S6-2 & 176.19 & -2.10 & 116 & 1.12 & $10.78 \pm 0.77$ & $1.87 \pm 0.51$ & $7.7 \times 10^{21}$ & & \\
\hline S6-2 & 176.19 & -2.10 & 74 & 0.72 & $10.66 \pm 0.62$ & $1.76 \pm 0.43$ & $8.0 \times 10^{21}$ & $5.4 \times 10^{3}$ & 60.0 \\
\hline S7-2 & 161.57 & -9.31 & 540 & 0.92 & $13.63 \pm 0.47$ & $2.07 \pm 0.12$ & $1.5 \times 10^{21}$ & & \\
\hline S7-4 & 161.57 & -9.32 & 116 & 0.20 & $13.35 \pm 1.13$ & $1.48 \pm 0.41$ & $2.4 \times 10^{21}$ & & \\
\hline S7-4 & 161.57 & -9.32 & 74 & 0.13 & $14.21 \pm 1.12$ & $1.19 \pm 0.30$ & $1.9 \times 10^{21}$ & $7.5 \times 10^{3}$ & 0.45 \\
\hline S8-2 & 109.81 & 2.70 & 74 & 0.29 & $21.02 \pm 2.81$ & $1.29 \pm 0.45$ & $5.9 \times 10^{21}$ & $1.0 \times 10^{4}$ & 7.2 \\
\hline S9-2 & 107.18 & 5.43 & 116 & 0.45 & $19.34 \pm 2.79$ & $1.44 \pm 0.51$ & $6.6 \times 10^{21}$ & $7.1 \times 10^{3}$ & 19.5 \\
\hline S10-1 & 300.88 & -8.94 & 360 & 0.39 & $11.84 \pm 0.65$ & $2.25 \pm 0.34$ & $5.4 \times 10^{21}$ & $6.7 \times 10^{3}$ & 12.2 \\
\hline
\end{tabular}

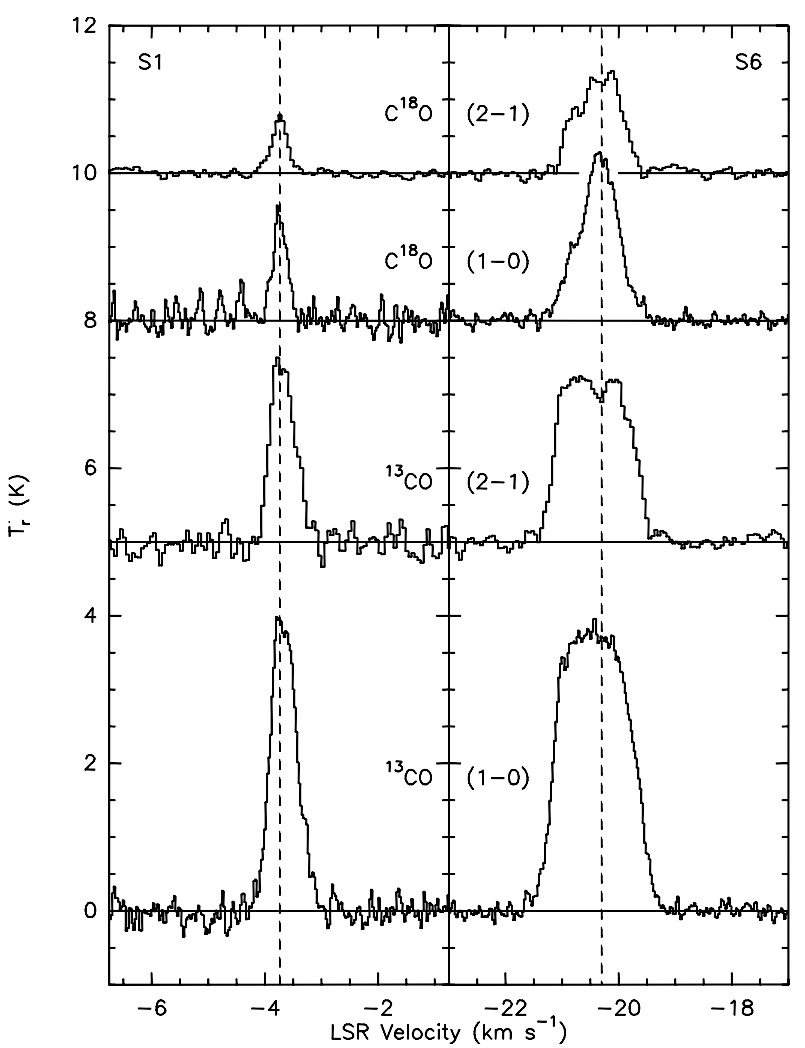

Fig. 7. CO spectral lines from the Planck clumps S1 and S6 (taken towards the central positions given in Table 1, and with beam apertures in Table A.1. The spectra are in the antenna temperature scale $\left(T_{\mathrm{a}}^{*}\right)$.

corresponding to a diameter of $74^{\prime \prime}$. The column densities are close to the values derived earlier (Table 3 ) from the dust continuum data, using the smaller aperture sizes (i.e., 74" or 116"). The estimated average densities of the cores are only of the order of $10^{3} \mathrm{~cm}^{-3}$, which is lower than the densities found in nearby dense cores (e.g., Myers et al. 1983). The reason for this is that

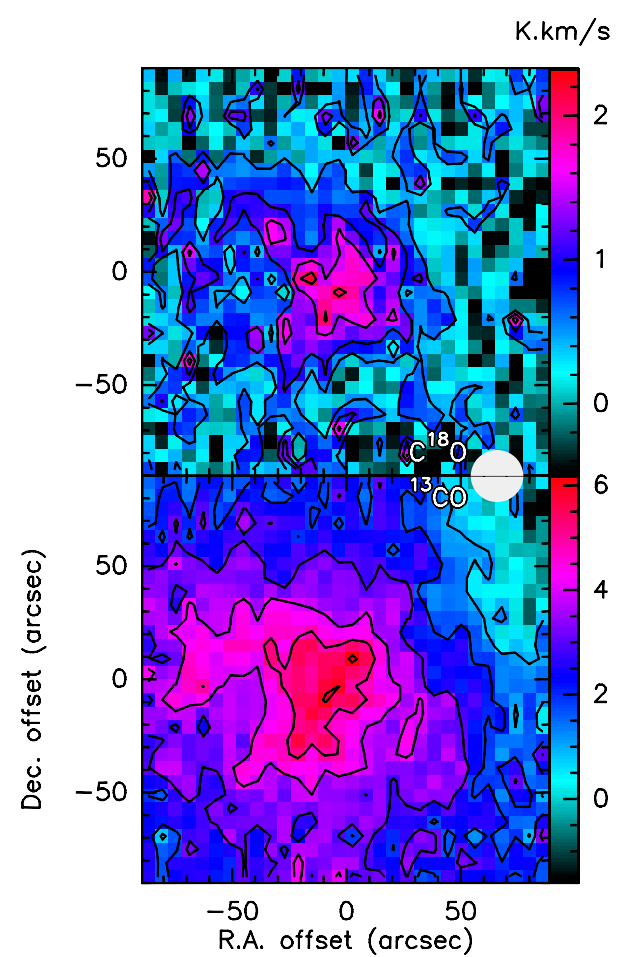

Fig. 8. The $\mathrm{S} 6$ source spectral line intensity maps for $\mathrm{C}^{18} \mathrm{O} J=1 \rightarrow 0$ (contour steps: $0.5 \mathrm{~K} \mathrm{~km} \mathrm{~s}^{-1}$, top image) and ${ }^{13} \mathrm{CO} \mathrm{J}=1 \rightarrow 0$ (contour steps: $1 \mathrm{~K} \mathrm{~km} \mathrm{~s}^{-1}$, bottom image). The white disk indicates the $30-\mathrm{m}$ HPBW size at the $2.7 \mathrm{~mm}$ wavelength, valid for both maps.

$\mathrm{C}^{18} \mathrm{O}$ is not a good tracer of dense gas because of the significant degree of $\mathrm{CO}$ depletion onto dust grains. Within a cold core, the depletion could be almost complete (e.g., Pineda et al. 2010) and so the investigation of this phenomenon towards the Planck cores is a natural topic for follow-up studies. 
Table 4. CO line parameters for selected lines-of-sight in seven cold core fields.

\begin{tabular}{|c|c|c|c|c|c|}
\hline Target & Telescope & Line & $\begin{array}{l}\text { FWHM } \\
\left(\mathrm{km} \mathrm{s}^{-1}\right)\end{array}$ & $\begin{array}{c}n_{\mathrm{H}_{2}}{ }^{a} \\
\left(\mathrm{~cm}^{-3}\right)\end{array}$ & $\begin{array}{l}N_{\mathrm{H}_{2}}{ }^{b} \\
\left(\mathrm{~cm}^{-2}\right)\end{array}$ \\
\hline \multirow[t]{6}{*}{$\overline{\mathrm{S} 1}$} & IRAM & ${ }^{12} \mathrm{CO} J=1 \rightarrow 0$ & $0.71 \pm 0.04$ & & \\
\hline & IRAM & ${ }^{12} \mathrm{CO} J=2 \rightarrow 1$ & $0.78 \pm 0.07$ & & \\
\hline & IRAM & ${ }^{13} \mathrm{CO} J=1 \rightarrow 0$ & $0.53 \pm 0.01$ & 3000 & $3.0 \times 10^{21}$ \\
\hline & IRAM & ${ }^{13} \mathrm{CO} J=2 \rightarrow 1$ & $0.50 \pm 0.02$ & & \\
\hline & IRAM & $\mathrm{C}^{18} \mathrm{O} J=1 \rightarrow 0$ & $0.30 \pm 0.02$ & 2700 & $4.0 \times 10^{21}$ \\
\hline & IRAM & $\mathrm{C}^{18} \mathrm{O} J=2 \rightarrow 1$ & $0.32 \pm 0.01$ & & \\
\hline \multirow[t]{3}{*}{ S3 } & Onsala & ${ }^{12} \mathrm{CO} J=1 \rightarrow 0$ & $2.64 \pm 0.03$ & & \\
\hline & Onsala & ${ }^{13} \mathrm{CO} J=1 \rightarrow 0$ & $1.71 \pm 0.04$ & & \\
\hline & Onsala & $\mathrm{C}^{18} \mathrm{O} J=1 \rightarrow 0$ & $1.22 \pm 0.08$ & 19000 & $6.7 \times 10^{21}$ \\
\hline \multirow[t]{4}{*}{ S6 } & IRAM & ${ }^{13} \mathrm{CO} J=1 \rightarrow 0$ & $1.44 \pm 0.02$ & & \\
\hline & IRAM & ${ }^{13} \mathrm{CO} J=2 \rightarrow 1$ & $1.42 \pm 0.02$ & & \\
\hline & IRAM & $\mathrm{C}^{18} \mathrm{O} J=1 \rightarrow 0$ & $0.82 \pm 0.01$ & 5000 & $1.4 \times 10^{22}$ \\
\hline & IRAM & $\mathrm{C}^{18} \mathrm{O} J=2 \rightarrow 1$ & $0.91 \pm 0.01$ & & \\
\hline \multirow[t]{4}{*}{$\mathrm{S} 7^{c}$} & IRAM & ${ }^{13} \mathrm{CO} J=1 \rightarrow 0$ & $1.60 \pm 0.10$ & & \\
\hline & IRAM & ${ }^{13} \mathrm{CO} J=2 \rightarrow 1$ & $1.70 \pm 0.10$ & & \\
\hline & IRAM & $\mathrm{C}^{18} \mathrm{O} J=1 \rightarrow 0$ & $1.40 \pm 0.20$ & 7300 & $5.6 \times 10^{21}$ \\
\hline & IRAM & $\mathrm{C}^{18} \mathrm{O} J=2 \rightarrow 1$ & $1.60 \pm 0.20$ & & \\
\hline \multirow[t]{2}{*}{ S8 } & Onsala & ${ }^{13} \mathrm{CO} J=1 \rightarrow 0$ & $2.06 \pm 0.07$ & & \\
\hline & Onsala & $\mathrm{C}^{18} \mathrm{O} J=1 \rightarrow 0$ & $1.82 \pm 1.07$ & 6400 & $1.3 \times 10^{22}$ \\
\hline \multirow[t]{2}{*}{ S9A } & Onsala & ${ }^{13} \mathrm{CO} J=1 \rightarrow 0$ & $2.54 \pm 0.04$ & & \\
\hline & Onsala & $\mathrm{C}^{18} \mathrm{O} J=1 \rightarrow 0$ & $1.57 \pm 0.12$ & 25000 & $1.8 \times 10^{22}$ \\
\hline \multirow[t]{2}{*}{ S9B } & Onsala & ${ }^{13} \mathrm{CO} J=1 \rightarrow 0$ & $1.95 \pm 0.05$ & & \\
\hline & Onsala & $\mathrm{C}^{18} \mathrm{O} J=1 \rightarrow 0$ & $1.41 \pm 0.11$ & 23000 & $1.6 \times 10^{22}$ \\
\hline \multirow[t]{3}{*}{$\mathrm{S} 10$} & APEX & ${ }^{12} \mathrm{CO} J=2 \rightarrow 1$ & $1.39 \pm 0.02$ & & \\
\hline & APEX & ${ }^{13} \mathrm{CO} J=2 \rightarrow 1$ & $0.82 \pm 0.03$ & & \\
\hline & APEX & $\mathrm{C}^{18} \mathrm{O} J=2 \rightarrow 1$ & $0.35 \pm 0.20$ & 23000 & $1.8 \times 10^{22}$ \\
\hline
\end{tabular}

Notes. ${ }^{a}$ Average density along the line of sight. ${ }^{b}$ Gas column density converted to $\mathrm{H}_{2}$ column density using the following conversion factors: $N_{\mathrm{H}_{2}}=10^{6} \times N\left({ }^{13} \mathrm{CO}\right)$; and $N_{\mathrm{H}_{2}}=10^{7} \times N\left(\mathrm{C}^{18} \mathrm{O}\right)$. ${ }^{c}$ For this source, the lines are an assembly of three components, with strongly asymmetric shapes, which are difficult to analyse separately.

\section{Discussion}

\subsection{The validity of the cold core detection method}

A perhaps unexpected result is the physical size of the Planck detections. They may have been anticipated to be compact point sources, the result of small cores being diluted within the large beam of Planck. However, they are instead found to be significantly extended and elongated, and embedded in filamentary (or cometary) larger-scale structures. As discussed in previous studies, the boundary of a core/clump/cloud structure is always difficult to assess. Curtis \& Richer (2010) for instance have shown how much it depends on the method used to identify and extract the core parameters.

In our case, the detection method used to extract sources from the Planck data is based on the colour signature of the objects, designed to enable us to detect the cold residuals after the removal of the warmer background. This results in the discovery of a different, more extended cold component with a more complex morphology than sources found with methods that identify structures on the basis of surface brightness (e.g., the "clumpfind" algorithm of Williams et al. (1994), or a multiscale wavelet analysis). The high sensitivity of Planck helps to better separate the warm and cold components, particularly in combination with data at somewhat shorter wavelengths.

The faint, mostly filamentary, emission of cold dust that we detect here could perhaps be called a "cold matrix" linking the substructures to each other over a broad range of scales. By combining Planck and Herschel it is possible to probe in detail the link between 10-pc scale filamentary structures and sub-parsec scale cold cores. Future studies with much larger samples should bring new insights to the origin of the cores and the mechanisms associated with their formation.

\subsection{The limitations of the SED fitting}

One has to be careful not to over-interpret SED fits based on a modified blackbody function, with a single colour temperature and optical depth. In reality the ISM has a broad distribution of dust temperatures and opacities (as well as column densities), within a Planck beam. Shetty et al. (2009) have discussed in detail the biases introduced by line-of-sight temperature variations and noise in the interpretation of such SED fits. Our study suggests that we are not badly contaminated by these effects for several reasons. Firstly Planck's broad spectral coverage, combined with IRIS allows us to determining both the dust temperature and emissivity spectral index. And secondly, we stress that the cold core detection method identifies structures where the submillimetre SED is dominated by localised dust emission colder than its large scale background.

An indirect indication that the parameters derived from the SEDs fits are not strongly biased is that the physical properties inferred cover the range of values known to be those of prestellar cores. Such cores have different properties whether they belong to regions of low- or high-mass star formation. Their column densities range between a few times $10^{21}$ or $10^{22} \mathrm{~cm}^{-2}$ in nearby star forming regions (Motte et al. 1998; Kauffmann et al. 2008; Enoch et al. 2006, 2007; Hatchell et al. 2005; Curtis \& Richer 2010; André et al. 2010) to $\sim 10^{23} \mathrm{~cm}^{-2}$ in the cores of infrared dark clouds (IRDCs, Simon et al. 2006; Rathborne et al. 2010; Peretto et al. 2010). These IRDCs, discovered by means of mid-infrared absorption towards the bright background emission of the Galactic Plane (MSX and ISOGAL surveys, Egan et al. 1998; Perault et al. 1996), are thought to be the sites of formation of massive stars and star clusters (Rathborne et al. 2006). The inferred average densities depend on their size, which ranges between $<0.1 \mathrm{pc}$ for low-mass dense cores of $\sim 1 M_{\odot}$, to $0.5 \mathrm{pc}$ or more for $\sim 10^{3} M_{\odot}$ IRDC cores. Our Planck cold clumps subsample is not located within the MSX IRDCs spatial distribution, so we cannot compare them directly with any IRDC association. However, the properties we derive fall well within this range. It is noteworthy that the Planck cold clumps are elongated structures which tend to exist within extended filaments (up to $30 \mathrm{pc}$ in the present subsample), with thickness up to $\sim 3$ pc. The IRDCs identified as extinction peaks in mid-infrared maps have similar lengthscales and column densities, but they are usually thinner and therefore have higher densities. It is also interesting that the linear mass densities of the filaments in which the Planck cold clumps are embedded cover the same range as the warm filamentary structures associated with active star-forming regions, i.e., extending up to several $100 M_{\odot} \mathrm{pc}^{-1}$.

The temperatures derived in the sample studied here cover a range $(10-14.5 \mathrm{~K})$ similar to previous estimates on a number of cold condensations detected using multi-wavelength submillimetre observations with the balloon-borne experiments: PRONAOS, $T \sim 12 \mathrm{~K}$ in star-forming and cirrus regions (Stepnik et al. 2003; Dupac et al. 2003; Bernard et al. 1999); Archeops, 7 $18 \mathrm{~K}$ (Désert et al. 2008); and BLAST, 9-14 K (Netterfield et al. 2009). These temperatures are all lower, however, than those measured in the so-called "quiescent" IRDC cores (Rathborne et al. 2010) that span from 17 to $30 \mathrm{~K}$ for a similar range in 
column density $\left(0.3-3 \times 10^{22} \mathrm{~cm}^{-2}\right)$ and mass $\left(10-10^{3} M_{\odot}\right)$. The Planck cold clump population may therefore be representative of a still earlier stage of evolution of cold dense cores.

\section{Summary and perspectives}

We have presented a preliminary analysis of a sample of 10 sources from the C3PO catalogue in order to illustrate and better probe the nature and properties of the cold objects detected with Planck. The sources have been chosen to span a broad range in temperature, density, mass and morphology (inluding filaments and isolated/clustered structures) in a variety of environments, from star-forming regions (both remote and nearby), to high Galactic latitude cirrus clouds. The main findings are as follows:

1. The sources are significantly larger than the Planck beam, with elongated shapes, and appear to belong to filamentary structures, with lengths up to $20 \mathrm{pc}$;

2. The physical parameters of the sources have been derived from SEDs by combining Planck (HFI bands at 857 , 545 , and $353 \mathrm{GHz}$ ) and IRIS data, finding $T \sim 10-15 \mathrm{~K}$ (with a mean value of $12.4 \mathrm{~K}$ ), $\beta \sim 1.8-2.5$ (mean 2.2) and $N_{\mathrm{H}_{2}} \sim 0.8-16 \times 10^{21} \mathrm{~cm}^{-2}$, from which we infer linear mass densities in the range $m=15-400 M_{\odot} \mathrm{pc}^{-1}$, masses $M \sim 3.5-1800 \mathrm{M}_{\odot}$, bolometric luminosities $L \sim 1-$ $300 L_{\odot}$, and $L / M \sim 0.1-0.9 L_{\odot} / M_{\odot}$;

3. Except for the faintest source (which lies at high Galactic latitude), a clear signature of cold dust emission is visible directly in the $1^{\circ} \times 1^{\circ}$ maps of dust temperature, spectral index and column density, with colour temperatures of typically $\sim 14 \mathrm{~K}$, surrounded by a warmer extended (often elongated) emission at around $16-18 \mathrm{~K}$;

4. Herschel and AKARI observations at higher angular resolution have revealed a rich and complex substructure within the Planck clumps, in most cases the substructures being colder (down to $7 \mathrm{~K}$ ) than the Planck-detected clumps, although in two cases, the substructures are warmer because they harbour compact infrared objects, likely protostellar sources at an early stage;

5. Molecular line observations of 7 of the sources show that all of them are clearly detected in ${ }^{13} \mathrm{CO}$ and $\mathrm{C}^{18} \mathrm{O},{ }^{13} \mathrm{CO}$ linewidths typically $1-2 \mathrm{~km} \mathrm{~s}^{-1}$, and $\mathrm{C}^{18} \mathrm{O}$ lines always narrower (down to $\sim 0.3 \mathrm{~km} \mathrm{~s}^{-1}$ ) but still clearly suprathermal, given the anticipated low temperature of the gas, suggesting that the support of Planck cold clumps against self-gravity is dominated by non-thermal motions.

Although we have focussed here on a very small sample of 10 clumps, the results are indicative of what might be expected from the more ambitious studies which will follow. We have already shown that the C3PO list of Planck cold sources contains objects with a wide variety of physical properties. These are probably associated with different evolutionary stages of the star formation process, from quiescent, cold and starless clumps, through prestellar stages to very young protostellar objects still embedded in their cold surrounding cloud. Forthcoming papers will present a more detailed analysis of the sources using Herschel data combined with Planck for larger and more statistically robust samples. More detailed physical modelling will allow for characterisation of clustering and fragmentation within the Planck clumps, addressing the question of their evolutionary stage, along with the study of stability of the starless substructures.
The first unbiased all-sky catalogue of cold objects provided by Planck offers the opportunity to investigate the properties of the population of Galactic cold objects over the entire sky. The full sample will include objects at the very early stages of evolution, in a variety of large-scale environments, and in particular, outside the well-known molecular complexes, at high latitude or at large distances within the Galactic Plane. For this purpose, dedicated follow-up observations are needed in both higher resolution continuum mode and spectroscopy, which is the objective of the "Galactic Cold Cores" key programme, planning a followup with PACS and SPIRE of about 300 Planck cold clumps. In parallel, similar and/or complementary follow-up studies will be possible on the basis of the ECC catalogue which has been delivered to the astronomical community, providing a robust subsample of the C3PO catalogue, with more than 900 Planck cold clumps distributed over the whole sky.

Acknowledgements. A description of the Planck Collaboration and a list of its members can be found at http://www.rssd.esa.int/index.php? project $=$ PLANCK\&page $=\mathrm{Pl}$ lanck_Collaboration. The Planck Collaboration acknowledges the support of: ESA; CNES and CNRS/INSU-IN2P3-INP (France); ASI, CNR, and INAF (Italy); NASA and DoE (USA); STFC and UKSA (UK); CSIC, MICINN and JA (Spain); Tekes, AoF and CSC (Finland); DLR and MPG (Germany); CSA (Canada); DTU Space (Denmark); SER/SSO (Switzerland); RCN (Norway); SFI (Ireland); FCT/MCTES (Portugal); and DEISA (EU) This publication makes use of data products from the Two Micron All Sky Survey, which is a joint project of the University of Massachusetts and the Infrared Processing and Analysis Center/California Institute of Technology, funded by the National Aeronautics and Space Administration and the National Science Foundation. This research has made use of the SIMBAD database, operated at CDS, Strasbourg, France. This research is based on observations with AKARI, a JAXA project with the participation of ESA.

\section{References}

André, P., Men'shchikov, A., Bontemps, S., et al. 2010, A\&A, 518, L102 Beckwith, S. V. W., Sargent, A. I., Chini, R. S., \& Guesten, R. 1990, AJ, 99, 924 Bensch, F., Leuenhagen, U., Stutzki, J., \& Schieder, R. 2003, ApJ, 591, 1013 Bergin, E. A., \& Tafalla, M. 2007, ARA\&A, 45, 339

Bernard, J. P., Abergel, A., Ristorcelli, I., et al. 1999, A\&A, 347, 640 Bersanelli, M., Mandolesi, N., Butler, R. C., et al. 2010, A\&A, 520, A4 Boden, K., \& Heithausen, A. 1993, A\&A, 268, 255

Bontemps, S., André, P., Könyves, V., et al. 2010, A\&A, 518, L85 Boudet, N., Mutschke, H., Nayral, C., et al. 2005, ApJ, 633, 272 Boulanger, F., Abergel, A., Bernard, J., et al. 1996, A\&A, 312, 256 Bourke, T. L., Hyland, A. R., \& Robinson, G. 1995, MNRAS, 276, 1052 Cantalupo, C. M., Borrill, J. D., Jaffe, A. H., Kisner, T. S., \& Stompor, R. 2010, ApJS, 187, 212

Compiègne, M., Flagey, N., Noriega-Crespo, A., et al. 2010, ApJ, 724, L44 Coupeaud, A., Demyk, K., Meny, C., \& Nayral, C. 2011, in IAU Symp., 280, 138

Crutcher, R. M., Hakobian, N., \& Troland, T. H. 2009, ApJ, 692, 844 Curtis, E. I., \& Richer, J. S. 2010, MNRAS, 402, 603

Dame, T. M., Hartmann, D., \& Thaddeus, P. 2001, ApJ, 547, 792

Désert, F., Macías-Pérez, J. F., Mayet, F., et al. 2008, A\&A, 481, 411

di Francesco, J., Sadavoy, S., Motte, F., et al. 2010, A\&A, 518, L91

Dobashi, K., Uehara, H., Kandori, R., et al. 2005, PASJ, 57, 1

Doi, Y., Etxaluze Azkonaga, M., White, G., et al. 2009, in The Next-Generation Infrared Space Mission: SPICA, ed. A. M. Heras, B. M. Swinyard, K. G. Isaak, \& J. R. Goicoechea, 4018

Dupac, X., Bernard, J., Boudet, N., et al. 2003, A\&A, 404, L11

Egan, M. P., Shipman, R. F., Price, S. D., et al. 1998, ApJ, 494, L199 Enoch, M. L., Young, K. E., Glenn, J., et al. 2006, ApJ, 638, 293 Enoch, M. L., Glenn, J., Evans, II, N. J., et al. 2007, ApJ, 666, 982 Falgarone, E., Pety, J., \& Phillips, T. G. 2001, ApJ, 555, 178 Fiege, J. D., \& Pudritz, R. E. 2000, ApJ, 534, 291

Fukui, Y., Mizuno, N., Yamaguchi, R., et al. 1999, PASJ, 51, 745 Goldreich, P., \& Kwan, J. 1974, ApJ, 189, 441

Górski, K. M., Hivon, E., Banday, A. J., et al. 2005, ApJ, 622, 759

Goss, W. M., Manchester, R. N., Brooks, J. W., et al. 1980, MNRAS, 191, 533

Griffin, M. J., Abergel, A., Abreu, A., et al. 2010, A\&A, 518, L3 
Haikala, L. K., Juvela, M., Harju, J., et al. 2006, A\&A, 454, L71 Hatchell, J., Richer, J. S., Fuller, G. A., et al. 2005, A\&A, 440, 151 Heithausen, A., \& Thaddeus, P. 1990, ApJ, 353, L49

Henning, T., Michel, B., \& Stognienko, R. 1995, Planet. Space Sci., 43, 1333

Hily-Blant, P., Falgarone, E., Pineau Des Forêts, G., \& Phillips, T. G. 2004, Ap\&SS, 292, 285

Johnstone, D., Wilson, C. D., Moriarty-Schieven, G., et al. 2000, ApJ, 545, 327 Jordi, C., Trullols, E., \& Galadi-Enriquez, D. 1996, A\&A, 312, 499

Juvela, M., Ristorcelli, I., Montier, L. A., et al. 2010, A\&A, 518, L93

Juvela, M., Ristorcelli, I., Pelkonen, V.-M., et al. 2011, A\&A, 527, A111

Kauffmann, J., Bertoldi, F., Bourke, T. L., Evans, II, N. J., \& Lee, C. W. 2008, A\&A, 487, 993

Könyves, V., André, P., Men'shchikov, A., et al. 2010, A\&A, 518, L106

Kramer, C., Stutzki, J., Rohrig, R., \& Corneliussen, U. 1998, A\&A, 329, 249

Kruegel, E., \& Siebenmorgen, R. 1994, A\&A, 288, 929

Lamarre, J., Puget, J., Ade, P. A. R., et al. 2010, A\&A, 520, A9

Leahy, J. P., Bersanelli, M., D'Arcangelo, O., et al. 2010, A\&A, 520, A8

Liseau, R., Lorenzetti, D., Nisini, B., Spinoglio, L., \& Moneti, A. 1992, A\&A, 265,577

Lombardi, M., \& Alves, J. 2001, A\&A, 377, 1023

Lynds, B. T. 1965, ApJS, 12, 163

Maheswar, G., Manoj, P., \& Bhatt, H. C. 2004, MNRAS, 355, 1272

Malinen, J., Juvela, M., Collins, D. C., Lunttila, T., \& Padoan, P. 2011, A\&A, 530, A101

Mandolesi, N., Bersanelli, M., Butler, R. C., et al. 2010, A\&A, 520, A3

Marshall, D. J., Robin, A. C., Reylé, C., Schultheis, M., \& Picaud, S. 2006, A\&A, 453, 635

McKee, C. F., \& Ostriker, E. C. 2007, ARA\&A, 45, 565

Mennella, A., Butler, R. C., Curto, A., et al. 2011, A\&A, 536, A3

Men'shchikov, A., André, P., Didelon, P., et al. 2010, A\&A, 518, L103

Meny, C., Gromov, V., Boudet, N., et al. 2007, A\&A, 468, 171

Mitra, S., Rocha, G., Górski, K. M., et al. 2011, ApJS, 193, 5

Miville-Deschênes, M., \& Lagache, G. 2005, ApJS, 157, 302

Molinari, S., Swinyard, B., Bally, J., et al. 2010, A\&A, 518, L100

Montier, L. A., Pelkonen, V., Juvela, M., Ristorcelli, I., \& Marshall, D. J. 2010, A\&A, 522, A83

Motte, F., Andre, P., \& Neri, R. 1998, A\&A, 336, 150

Motte, F., Bontemps, S., Schilke, P., et al. 2007, A\&A, 476, 1243

Motte, F., Zavagno, A., Bontemps, S., et al. 2010, A\&A, 518, L77

Murakami, H., Baba, H., Barthel, P., et al. 2007, PASJ, 59, 369

Murphy, D. C., \& May, J. 1991, A\&A, 247, 202

Myers, P. C., Linke, R. A., \& Benson, P. J. 1983, ApJ, 264, 517

Netterfield, C. B., Ade, P. A. R., Bock, J. J., et al. 2009, ApJ, 707, 1824

Obayashi, A., Kun, M., Sato, F., Yonekura, Y., \& Fukui, Y. 1998, AJ, 115, 274

Olmi, L., Ade, P. A. R., Anglés-Alcázar, D., et al. 2009, ApJ, 707, 1836

Ossenkopf, V., \& Henning, T. 1994, A\&A, 291, 943

Otrupcek, R. E., Hartley, M., \& Wang, J. 2000, PASA, 17, 92

Pagani, L., Daniel, F., \& Dubernet, M. 2009, A\&A, 494, 719

Perault, M., Omont, A., Simon, G., et al. 1996, A\&A, 315, L165

Peretto, N., Fuller, G. A., Plume, R., et al. 2010, A\&A, 518, L98

Pilbratt, G. L., Riedinger, J. R., Passvogel, T., et al. 2010, A\&A, 518, L1

Pineda, J. L., Goldsmith, P. F., Chapman, N., et al. 2010, ApJ, 721, 686

Planck Collaboration 2011a, A\&A, 536, A1

Planck Collaboration 2011b, A\&A, 536, A2

Planck Collaboration 2011c, A\&A, 536, A7

Planck Collaboration 2011d, A\&A, 536, A8

Planck Collaboration 2011e, A\&A, 536, A9

Planck Collaboration 2011f, A\&A, 536, A10

Planck Collaboration 2011g, A\&A, 536, A11

Planck Collaboration 2011h, A\&A, 536, A12

Planck Collaboration 2011i, A\&A, 536, A13

Planck Collaboration. 2011j, A\&A, 536, A14

Planck Collaboration 2011k, A\&A, 536, A15

Planck Collaboration 20111, A\&A, 536, A16

Planck Collaboration 2011m, A\&A, 536, A17

Planck Collaboration 2011n, A\&A, 536, A18

Planck Collaboration 2011o, A\&A, 536, A19

Planck Collaboration 2011p, A\&A, 536, A20

Planck Collaboration 2011q, A\&A, 536, A21

Planck Collaboration 2011r, A\&A, 536, A22

Planck Collaboration 2011s, A\&A, 536, A23

Planck Collaboration 2011t, A\&A, 536, A24

Planck Collaboration 2011u, A\&A, 536, A25

Planck Collaboration 2011v, The Explanatory Supplement to the Planck Early

Release Compact Source Catalogue (ESA)

Planck Collaboration 2011w, A\&A, 536, A26

Planck HFI Core Team 2011a, A\&A, 536, A4

Planck HFI Core Team 2011b, A\&A, 536, A6
Poglitsch, A., Waelkens, C., Geis, N., et al. 2010, A\&A, 518, L2

Preibisch, T., Ossenkopf, V., Yorke, H. W., \& Henning, T. 1993, A\&A, 279, 577

Rathborne, J. M., Jackson, J. M., \& Simon, R. 2006, ApJ, 641, 389

Rathborne, J. M., Jackson, J. M., Chambers, E. T., et al. 2010, ApJ, 715, 310

Rosset, C., Tristram, M., Ponthieu, N., et al. 2010, A\&A, 520, A13

Roy, A., Ade, P. A. R., Bock, J. J., et al. 2011, ApJ, 730, 142

Sargent, A. I. 1977, ApJ, 218, 736

Schlegel, D. J., Finkbeiner, D. P., \& Davis, M. 1998, ApJ, 500, 525

Schneider, N., Motte, F., Bontemps, S., et al. 2010, A\&A, 518, L83

Shetty, R., Kauffmann, J., Schnee, S., Goodman, A. A., \& Ercolano, B. 2009, ApJ, 696, 2234

Simon, R., Rathborne, J. M., Shah, R. Y., Jackson, J. M., \& Chambers, E. T. 2006, ApJ, 653, 1325

Skrutskie, M., Cutri, R., Stiening, R., et al. 2006, AJ, 131, 1163, owner: marshall Added to JabRef: 2008.07.29

Stepnik, B., Abergel, A., Bernard, J., et al. 2003, A\&A, 398, 551

Stickel, M., Krause, O., Klaas, U., \& Lemke, D. 2007, A\&A, 466, 1205

Stutz, A., Launhardt, R., Linz, H., et al. 2010, A\&A, 518, L87

Tafalla, M., Bachiller, R., \& Martin-Pintado, J. 1993, ApJ, 403, 175

Tauber, J. A., Mandolesi, N., Puget, J., et al. 2010, A\&A, 520, A1

Ungerechts, H., \& Thaddeus, P. 1987, ApJS, 63, 645

van Till, H., Loren, R., \& Davis, J. 1975, ApJ, 198, 235

Vilas-Boas, J. W. S., Myers, P. C., \& Fuller, G. A. 1994, ApJ, 433, 96

Ward-Thompson, D., André, P., Crutcher, R., et al. 2007, Protostars and Planets $\mathrm{V}, 33$

Ward-Thompson, D., Kirk, J. M., André, P., et al. 2010, A\&A, 518, L92

White, G. J. 1993, A\&A, 274, L33

Williams, J. P., de Geus, E. J., \& Blitz, L. 1994, ApJ, 428, 693

Williams, J. P., Blitz, L., \& McKee, C. F. 2000, Protostars and Planets IV, 97

Yamamura, I. 2008, in COSPAR, Plenary Meeting, 37th COSPAR Scientific Assembly, 37, 3529

Yonekura, Y., Mizuno, N., Saito, H., et al. 1999, PASJ, 51, 911

Zacchei, A., Maino, D., Baccigalupi, C., et al. 2011, A\&A, 536, A5

Zavagno, A., Russeil, D., Motte, F., et al. 2010, A\&A, 518, L81

1 Aalto University Metsähovi Radio Observatory, Metsähovintie 114, 02540 Kylmälä, Finland

2 Agenzia Spaziale Italiana Science Data Center, c/o ESRIN, Via Galileo Galilei, Frascati, Italy

3 Astroparticule et Cosmologie, CNRS (UMR7164), Université Denis Diderot Paris 7, Bâtiment Condorcet, 10 rue A. Domon et Léonie Duquet, Paris, France

4 Astrophysics Group, Cavendish Laboratory, University of Cambridge, JJ Thomson Avenue, Cambridge CB3 0HE, UK

5 Atacama Large Millimeter/submillimeter Array, ALMA Santiago Central Offices, Alonso de Cordova 3107, Vitacura, Casilla 763 0355, Santiago, Chile

6 CITA, University of Toronto, 60 St. George St., Toronto, ON M5S 3H8, Canada

7 CNRS, IRAP, 9 Av. colonel Roche, BP 44346, 31028 Toulouse Cedex 4, France

8 California Institute of Technology, Pasadena, California, USA

9 DAMTP, University of Cambridge, Centre for Mathematical Sciences, Wilberforce Road, Cambridge CB3 0WA, UK

10 DSM/Irfu/SPP, CEA-Saclay, 91191 Gif-sur-Yvette Cedex, France

11 DTU Space, National Space Institute, Juliane Mariesvej 30, Copenhagen, Denmark

12 Departamento de Física, Universidad de Oviedo, Avda. Calvo Sotelo s/n, Oviedo, Spain

13 Department of Astronomy and Astrophysics, University of Toronto, 50 Saint George Street, Toronto, Ontario, Canada

14 Department of Earth Sciences and Astronomy, University of Tokyo, Komaba 3-8-1, Meguro, Tokyo 153-8902, Japan

15 Department of Physics \& Astronomy, University of British Columbia, 6224 Agricultural Road, Vancouver, British Columbia, Canada

16 Department of Physics, Gustaf Hällströmin katu 2a, University of Helsinki, Helsinki, Finland

17 Department of Physics, Princeton University, Princeton, New Jersey, USA

18 Department of Physics, Purdue University, 525 Northwestern Avenue, West Lafayette, Indiana, USA 
19 Department of Physics, University of California, Berkeley, California, USA

20 Department of Physics, University of California, One Shields Avenue, Davis, California, USA

21 Department of Physics, University of California, Santa Barbara, California, USA

22 Department of Physics, University of Illinois at Urbana-Champaign, 1110 West Green Street, Urbana, Illinois, USA

23 Dipartimento di Fisica G. Galilei, Università degli Studi di Padova, Via Marzolo 8, 35131 Padova, Italy

24 Dipartimento di Fisica, Università La Sapienza, P.le A. Moro 2, Roma, Italy

25 Dipartimento di Fisica, Università degli Studi di Milano, Via Celoria, 16, Milano, Italy

26 Dipartimento di Fisica, Università degli Studi di Trieste, Via A. Valerio 2, Trieste, Italy

27 Dipartimento di Fisica, Università di Ferrara, Via Saragat 1, 44122 Ferrara, Italy

28 Dipartimento di Fisica, Università di Roma Tor Vergata, Via della Ricerca Scientifica 1, Roma, Italy

29 Discovery Center, Niels Bohr Institute, Blegdamsvej 17, Copenhagen, Denmark

30 Dpto. Astrofísica, Universidad de La Laguna (ULL), 38206 La Laguna, Tenerife, Spain

31 Eötvös Loránd University, Department of Astronomy, Pázmány Péter sétány 1/A, 1117 Budapest, Hungary

32 European Southern Observatory, ESO Vitacura, Alonso de Cordova 3107, Vitacura, Casilla 19001, Santiago, Chile

33 European Space Agency, ESAC, Planck Science Office, Camino bajo del Castillo s/n, Urbanización Villafranca del Castillo, Villanueva de la Cañada, Madrid, Spain

34 European Space Agency, ESTEC, Keplerlaan 1, 2201 AZ Noordwijk, The Netherlands

35 Helsinki Institute of Physics, Gustaf Hällströmin katu 2, University of Helsinki, Helsinki, Finland

${ }^{36}$ INAF - Osservatorio Astrofisico di Catania, Via S. Sofia 78, Catania, Italy

37 INAF - Osservatorio Astronomico di Padova, Vicolo dell'Osservatorio 5, Padova, Italy

38 INAF - Osservatorio Astronomico di Roma, Via di Frascati 33, Monte Porzio Catone, Italy

39 INAF - Osservatorio Astronomico di Trieste, Via G.B. Tiepolo 11, Trieste, Italy

${ }^{40}$ INAF/IASF Bologna, Via Gobetti 101, Bologna, Italy

41 INAF/IASF Milano, Via E. Bassini 15, Milano, Italy

42 INRIA, Laboratoire de Recherche en Informatique, Université Paris-Sud 11, Bâtiment 490, 91405 Orsay Cedex, France

43 IPAG (Institut de Planétologie et d'Astrophysique de Grenoble), Université Joseph Fourier, Grenoble 1/CNRS-INSU, UMR 5274, 38041 Grenoble, France

44 Imperial College London, Astrophysics group, Blackett Laboratory, Prince Consort Road, London, SW7 2AZ, UK

45 Infrared Processing and Analysis Center, California Institute of Technology, Pasadena, CA 91125, USA

46 Institut Néel, CNRS, Université Joseph Fourier Grenoble I, 25 rue des Martyrs, Grenoble, France

47 Institut d'Astrophysique Spatiale, CNRS (UMR8617) Université Paris-Sud 11, Bâtiment 121, Orsay, France

48 Institut d'Astrophysique de Paris, CNRS UMR7095, Université Pierre \& Marie Curie, 98bis boulevard Arago, Paris, France

49 Institute of Astronomy and Astrophysics, Academia Sinica, Taipei, Taiwan

50 Institute of Astronomy, University of Cambridge, Madingley Road, Cambridge CB3 OHA, UK
51 Institute of Space and Astronautical Science, Japan Aerospace Exploration Agency, 3-1-1 Yoshinodai, Chuo-ku, Sagamihara, Kanagawa, 252-5210, Japan

52 Institute of Theoretical Astrophysics, University of Oslo, Blindern, Oslo, Norway

53 Instituto de Astrofísica de Canarias, C/Vía Láctea s/n, La Laguna, Tenerife, Spain

54 Instituto de Física de Cantabria (CSIC-Universidad de Cantabria), Avda. de los Castros s/n, Santander, Spain

55 Jet Propulsion Laboratory, California Institute of Technology, 4800 Oak Grove Drive, Pasadena, California, USA

56 Jodrell Bank Centre for Astrophysics, Alan Turing Building, School of Physics and Astronomy, The University of Manchester, Oxford Road, Manchester M13 9PL, UK

57 Kavli Institute for Cosmology Cambridge, Madingley Road, Cambridge CB3 OHA, UK

58 LERMA, CNRS, Observatoire de Paris, 61 avenue de l'Observatoire, Paris, France

59 Laboratoire AIM, IRFU/Service d'Astrophysique - CEA/DSM CNRS - Université Paris Diderot, Bât. 709, CEA-Saclay, 91191 Gifsur-Yvette Cedex, France

60 Laboratoire Traitement et Communication de l'Information, CNRS (UMR 5141) and Télécom ParisTech, 46 rue Barrault, 75634 Paris Cedex 13, France

${ }^{61}$ Laboratoire de Physique Subatomique et de Cosmologie, CNRS/IN2P3, Université Joseph Fourier Grenoble I, Institut National Polytechnique de Grenoble, 53 rue des Martyrs, 38026 Grenoble Cedex, France

${ }^{62}$ Laboratoire de l'Accélérateur Linéaire, Université Paris-Sud 11, CNRS/IN2P3, Orsay, France

${ }^{63}$ Lawrence Berkeley National Laboratory, Berkeley, California, USA

${ }^{64}$ Max-Planck-Institut für Astrophysik, Karl-Schwarzschild-Str. 1, 85741 Garching, Germany

${ }^{65}$ MilliLab, VTT Technical Research Centre of Finland, Tietotie 3, Espoo, Finland

${ }^{66}$ National University of Ireland, Department of Experimental Physics, Maynooth, Co. Kildare, Ireland

67 Niels Bohr Institute, Blegdamsvej 17, Copenhagen, Denmark

68 Observational Cosmology, Mail Stop 367-17, California Institute of Technology, Pasadena, CA, 91125, USA

69 Optical Science Laboratory, University College London, Gower Street, London, UK

70 SISSA, Astrophysics Sector, Via Bonomea 265, 34136 Trieste, Italy

71 SUPA, Institute for Astronomy, University of Edinburgh, Royal Observatory, Blackford Hill, Edinburgh EH9 3HJ, UK

72 School of Physics and Astronomy, Cardiff University, Queens Buildings, The Parade, Cardiff CF24 3AA, UK

73 Space Sciences Laboratory, University of California, Berkeley, California, USA

74 Spitzer Science Center, 1200 E. California Blvd., Pasadena, California, USA

75 Stanford University, Dept of Physics, Varian Physics Bldg, 382 Via Pueblo Mall, Stanford, California, USA

76 Université de Toulouse, UPS-OMP, IRAP, 31028 Toulouse Cedex 4, France

77 Universities Space Research Association, Stratospheric Observatory for Infrared Astronomy, MS 211-3, Moffett Field, CA 94035, USA

78 University of Granada, Departamento de Física Teórica y del Cosmos, Facultad de Ciencias, Granada, Spain

79 University of Miami, Knight Physics Building, 1320 Campo Sano Dr., Coral Gables, Florida, USA

${ }^{80}$ Warsaw University Observatory, Aleje Ujazdowskie 4, 00-478 Warszawa, Poland 


\section{Appendix A: Selected sources, illustration of detection method, source elongation and observational parameters}

We list below the main features of the selected fields as known based on previous studies.

S1 lies in a tenuous high-latitude cloud, MCLD 126.5+24.5, located at the border of the Polaris Flare, a large molecular cirrus cloud in the direction of the north celestial pole (Heithausen \& Thaddeus 1990), at an estimated distance of $150 \mathrm{pc}$ (Bensch et al. 2003). The cloud was first noted from the POSS prints by Lynds (1965) as the small reflection nebula LBN 628. Its cometary globule shape appears similar to what is usually found in active star formation regions, although this nebula is far from any such region. Boden \& Heithausen (1993) have studied the gas properties of the densest part of the cloud, observing transition lines of the dense tracors ${ }^{12} \mathrm{CO},{ }^{13} \mathrm{CO}, \mathrm{H}_{2} \mathrm{CO}$, and $\mathrm{NH}_{3}$. The ammonia core $(\sim 0.14 \mathrm{pc} \times 0.07 \mathrm{pc})$ corresponds to $0.2 M_{\odot}$ and a density of $4000 \mathrm{~cm}^{-3}$. Although this density is high compared to the cirrus-like environment, Boden \& Heithausen (1993) find that both the cloud and the core are probably gravitationally unbound. The line ratios found are consistent with shocked gas being compressed by the North Celestial Pole Hi loop (Kramer et al. 1998).

S2 is embedded in the tail ( $\sim 30^{\prime}$ south) of the well-studied cometary globule CG12. This isolated globule is located at high Galactic latitude $\left(l=316.5^{\circ}, b=21.2^{\circ}\right)$, with a $1^{\circ}$ long nebular tail nearly perpendicular to the Galactic Plane. Its distance of $550 \mathrm{pc}$ has been determined using a photometry-extinction method by Maheswar et al. (2004). Molecular studies using ${ }^{12}$ CO (van Till et al. 1975; White 1993; Yonekura et al. 1999), $\mathrm{C}^{18} \mathrm{O}$ (Haikala et al. 2006), $\mathrm{H}_{2} \mathrm{CO}$ (Goss et al. 1980) and $\mathrm{NH}_{3}$ (Bourke et al. 1995) reveal star formation activity, with the presence of a highly collimated bipolar outflow in the head of the cloud. CG12 seems to be a rare example of triggered star formation at relatively large Galactic height (Maheswar et al. 2004).

S3 is located in the constellation of Cassiopeia and has no counterpart in the SIMBAD database. On the sky, the closest Lynds catalogue sources are LDN 1358 and LDN 1355, which are, respectively, at distances of 114 and 118'. These Lynds sources are associated with the Cepheus flare at a distance of $200 \pm 50$ pc (Obayashi et al. 1998; Kauffmann et al. 2008). The Planck detection is associated with an AKARI FIS bright source catalogue source that has a cirrus type spectrum (Yamamura 2008).

S4 is in the Orion region, within a dark cloud that was mapped by Dobashi et al. (2005) in extinction, derived from Digitized Sky Survey images. The source is located six degrees east of the M42 nebula, in the Dobashi et al. cloud 1490, close to its clump P43. It has not been the subject of any dedicated studies, so far. In higher resolution extinction images derived from the colour excess of 2MASS stars, the cloud is seen to reside within a narrow filament. In the Planck data, the filament is clearly visible, but remains unresolved. The source itself is a compact, slightly elongated clump that stands out just as well in the individual Planck frequency maps as in the cold residual map. The Nanten $\mathrm{CO}$ data show a peak at the same position, with a line intensity close to $20 \mathrm{~K} \mathrm{~km} \mathrm{~s}^{-1}$.

S5 is a filamentary structure located near the Vela Molecular Ridge (hereafter VMR), a giant molecular cloud complex in the outer Galaxy. Murphy \& May (1991) mapped the VMR in $\mathrm{CO}$, but the mapping did not cover S5. However, Otrupcek et al. (2000) carried out CO observations of the dark cloud DCld $276.9+01.7$, with angular extent of $16^{\prime}$ by $3^{\prime}$, and this is interposed on the filament. The detected $\mathrm{CO}$ line is rather strong $\left(T_{\mathrm{A}}^{*}=6.1 \mathrm{~K}\right)$, with a velocity (relative to the local standard of rest) of $\sim 2 \mathrm{~km} \mathrm{~s}^{-1}$. We derive a kinematic distance for the cloud of $\sim 2 \mathrm{kpc}$. This is consistent with the distance estimate of the VMR: Liseau et al. (1992) give a photometric distance of $(0.7 \pm 0.2) \mathrm{kpc}$ for parts $\mathrm{A}, \mathrm{C}$ and $\mathrm{D}$ of the VMR and $2 \mathrm{kpc}$ for part B, using the nomenclature established by Murphy \& May (1991) and agreeing with their results. Thus we consider it is likely that S5 is part of the VMR. Several studies have been published on the VMR young star-forming region, for example, Olmi et al. (2009) found 141 BLAST cores (starless and protostellar) in the Vela-D region.

S6 has been detected in the anti-centre direction $(l, b=$ $176.18, b=-2.11)$, at a distance estimated to $2 \mathrm{kpc}$ using the extinction signature method by Marshall et al. (2006). The filament-shaped core seen with Planck does not coincide with any known object, but is likely associated with the Perseus arm.

S7 has been observed by Ungerechts \& Thaddeus (1987) as part of their large CO survey of Perseus, Taurus and Auriga. It is included in their 12th area (see their Table 1), covering $41.8 \mathrm{deg}^{2}$, with a distance of $350 \mathrm{pc}$. For this large region, they estimate a virial mass that is four times higher than the $\mathrm{CO}$ mass, showing that this region is out of equilibrium. The Planck source is not associated with any known objects in the SIMBAD database.

S8, S9 and S10 correspond with Herschel SDP sources PCC288, PCC550 and PCC249, respectively. These fields were selected in September 2009 from the cold core detections in the First Light Survey of Planck, in order to perform the first follow-up observations of our Herschel key-program during the Herschel SDP. The fields were mapped with both PACS and SPIRE (with map sizes from $18^{\prime}$ to $50^{\prime}$ ), and the observations have been described in detail in dedicated papers (Juvela et al. 2010, 2011).

Field S8 is located in Cepheus, at the interface between the Cepheus F molecular cloud (Sargent 1977) and the young stellar group Cep OB3b (Jordi et al. 1996), which is one of the youngest nearby stellar groups. It has been suggestsed that this region could show direct evidence for star formation triggered by the $\mathrm{OB}$ association. $\mathrm{S} 9$ is an active star formation region close the S140 H II region. Planck has provided two detections, PCC288P1 and PCC288-P2 (see Juvela et al. 2010), with the southern source PCC288-P2 being the colder one. The two cores have also been identified in CS $J=1 \rightarrow 0$ mapping of the area by Tafalla et al. (1993). The field S10 is part of the Musca cloud filament, where Planck shows two secure detections of cold cores $(T \sim 11 \mathrm{~K})$. The cores have been studied previously in ${ }^{13} \mathrm{CO}$ and $\mathrm{C}^{18} \mathrm{O}$ by Vilas-Boas et al. (1994). 
Planck Collaboration: Planck early results. XXII.

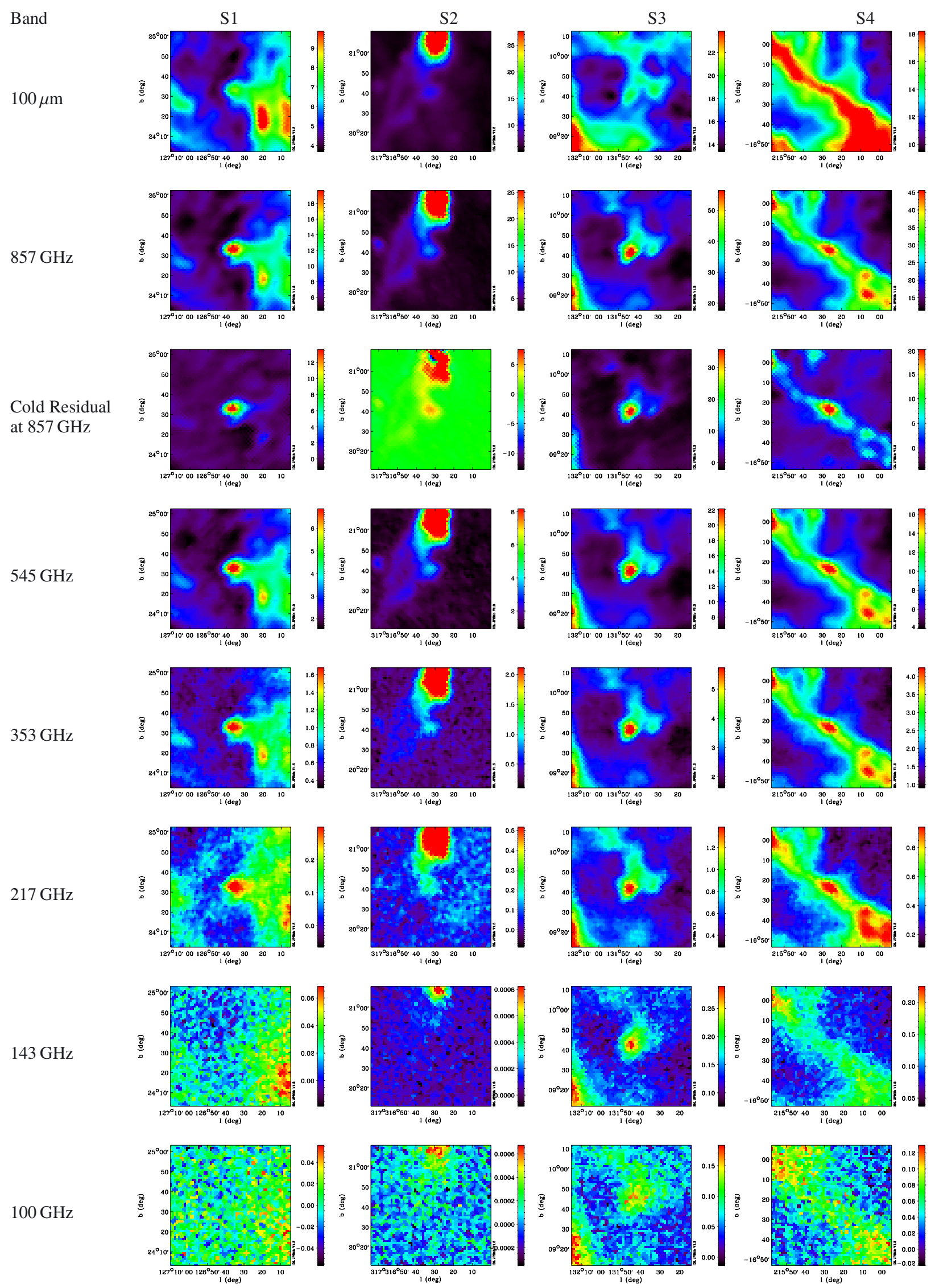

Fig. A.1. Multi-band emission maps (in MJy/sr) of the sources S1, S2, S3, and S4. 
A\&A 536, A22 (2011)

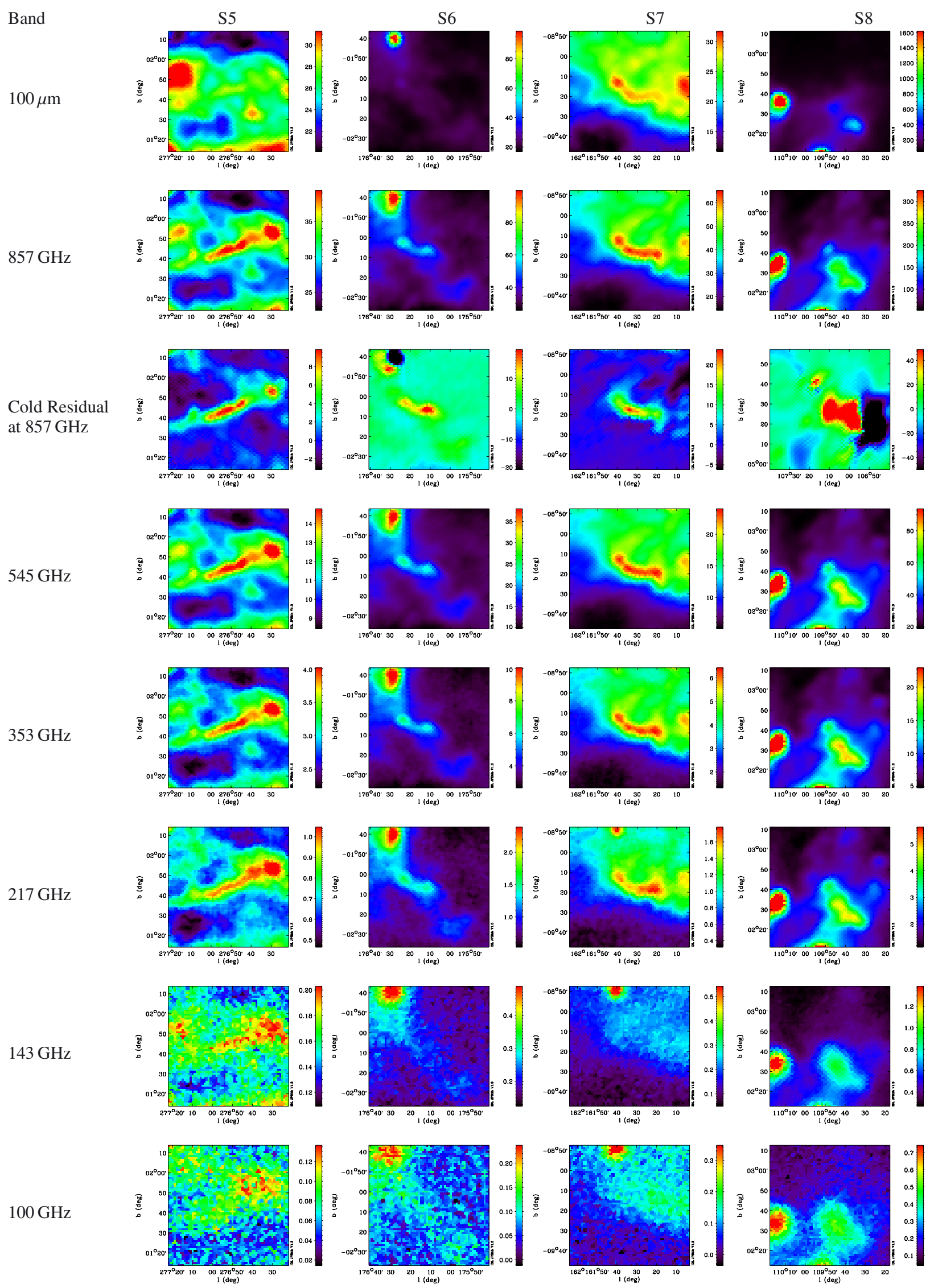

Fig. A.2. Multi-band emission maps (in MJy/sr) of the sources S5, S6, S7, and S8. 
Planck Collaboration: Planck early results. XXII.

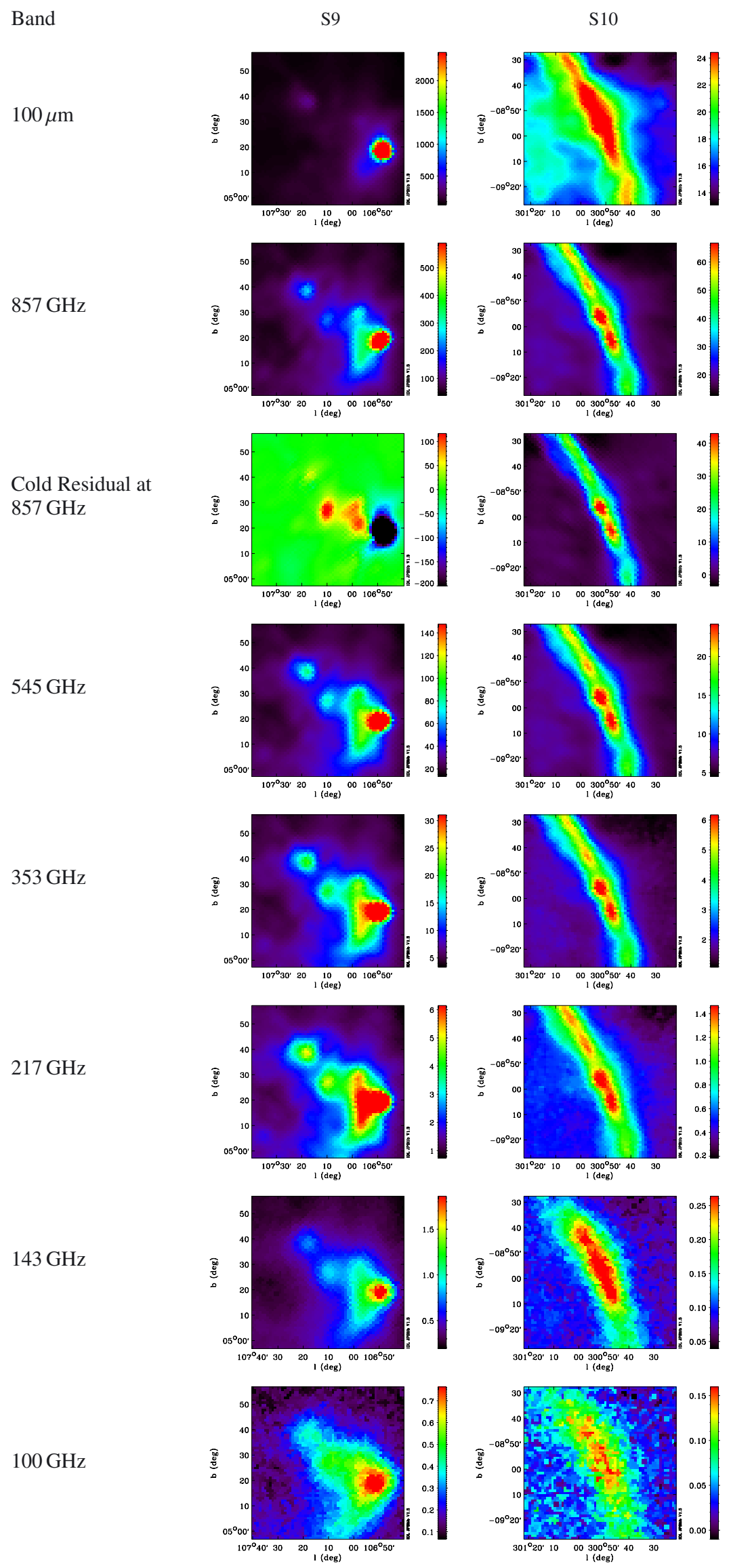

Fig. A.3. Multi-band emission maps (in MJy/sr) of the sources S9 and S10. 

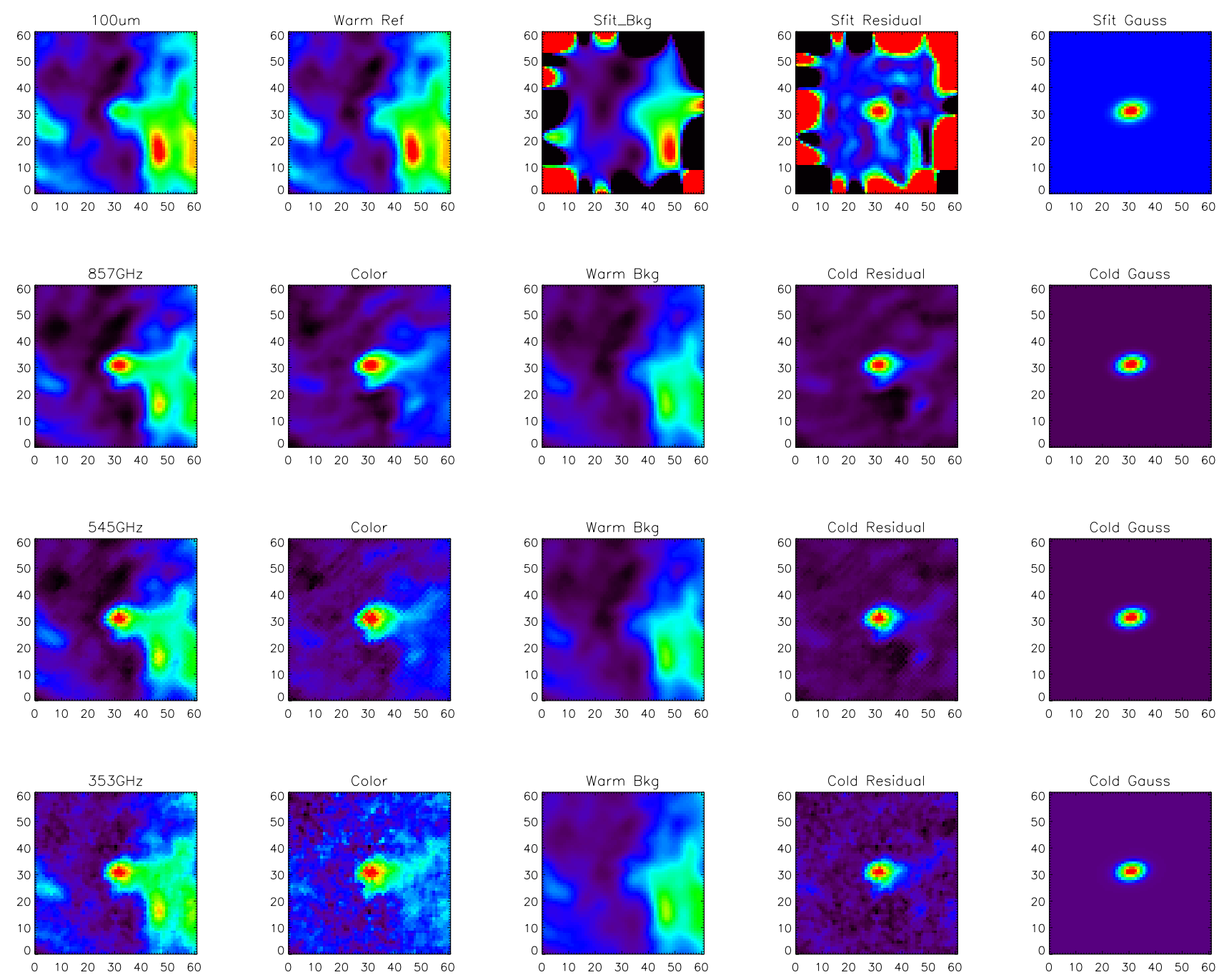

Fig. A.4. Illustration of the steps used in the detection and core extraction methods, as applied to source S1. The first column shows the initial surface brightness emission maps in the IRIS and HFI bands. The rows show the process applied to the $100 \mu \mathrm{m}, 857,545$, and $353 \mathrm{GHz}$ data, respectively. As described in Paper I, the method follows the following main steps: a "Warm Ref" is built from the IRIS $100 \mu \mathrm{m}$ map and extrapolated to the Planck bands using the local colour estimated around each pixel of the Planck map (these colour maps are shown in the second column); the "Warm Bkg" map obtained at a given frequency is then removed from the Planck map, revealing the "Cold Residual"; and finally an elliptical Gaussian fit is performed on the colour maps to derive the core size parameters, while aperture photometry is performed on the HFI bands, integrating the signal within the elliptical Gaussian. 
Planck Collaboration: Planck early results. XXII.

$\mathrm{S} 1$

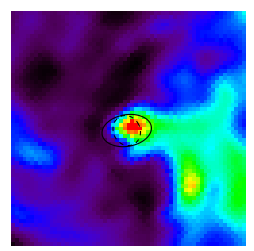

S3

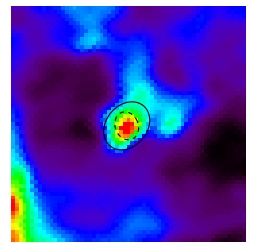

S5

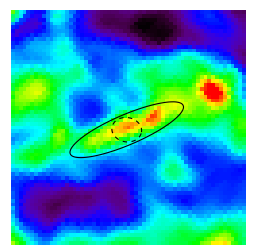

S7

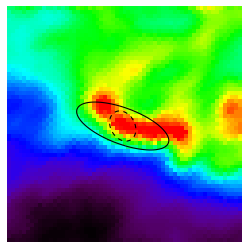

S9

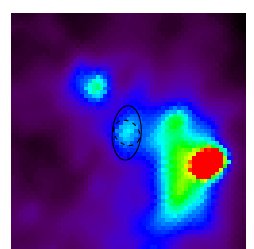

S2

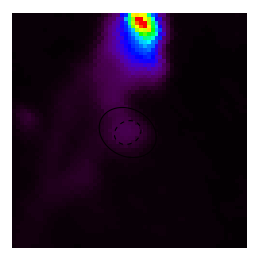

S4

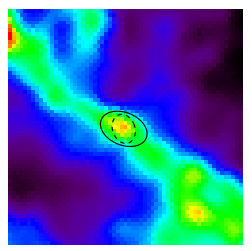

S6

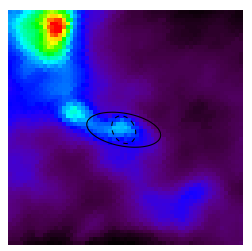

S8

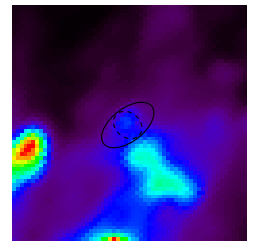

S10

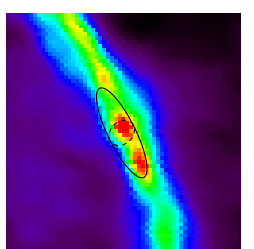

Fig. A.5. Comparison of the source elongation with the beam shape determined with the "FEBeCoP" tool (Mitra et al. 2011). The ellipses correspond to the size of the Planck cold clumps derived from the fit; they are plotted at a $2 \sigma$ level for a better visibility. The dashed circles trace the local beam shape.
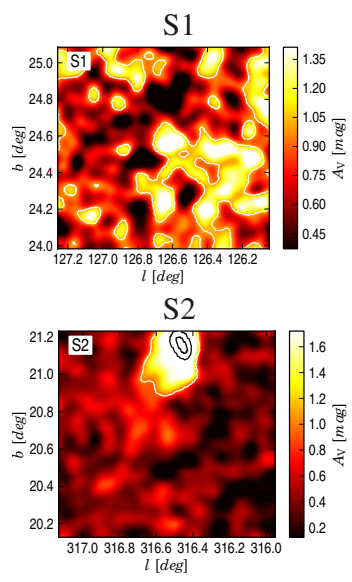

S3

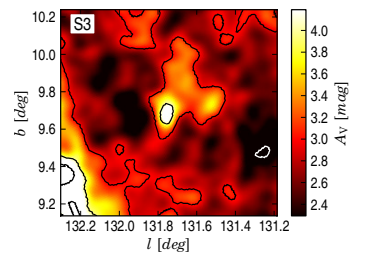

S4

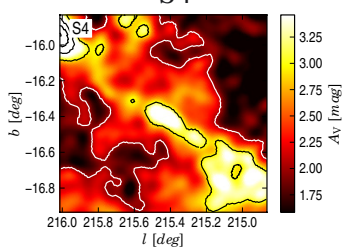

S5

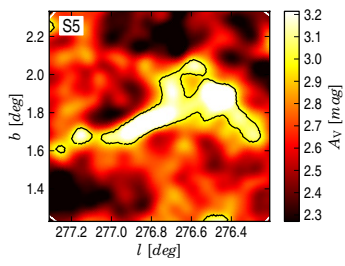

S6

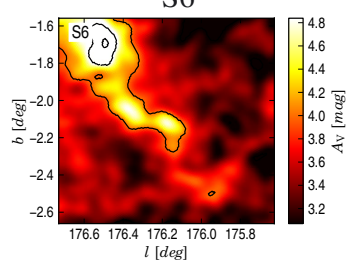

S7

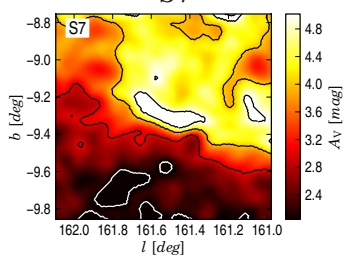

Fig. A.6. Visual extinction maps obtained from 2MASS and the NICER method (cf. Sect. 4.2). The maps are shown at $4.5^{\prime}$ resolution. Contours are drawn at integer values of $A_{V}$. 
Table A.1. Observational parameters and sensitivity of IRAM observations.

\begin{tabular}{lccccc}
\hline \hline Molecule & Transition & $\begin{array}{c}\text { Frequency } \\
(\mathrm{MHz})\end{array}$ & $\begin{array}{c}\text { Beam size } \\
(\operatorname{arcsec})\end{array}$ & $\begin{array}{c}\delta v \\
\left(\mathrm{~m} \mathrm{~s}^{-1}\right)\end{array}$ & $\begin{array}{c}\mathrm{rms} \\
(\mathrm{K})\end{array}$ \\
\hline 1 st setting & & & & & \\
\hline${ }^{12} \mathrm{CO}$ & $J=1 \rightarrow 0$ & 115271.197 & 23 & 200 & 0.24 \\
${ }^{12} \mathrm{CO}$ & $J=2 \rightarrow 1$ & 230537.990 & 12 & 100 & 0.15 \\
\hline${ }^{2 \mathrm{nd} \text { setting }}$ & & & & & \\
\hline${ }^{13} \mathrm{CO}$ & $J=1 \rightarrow 0$ & 110201.370 & 24 & 27 & 0.15 \\
${ }^{13} \mathrm{CO}$ & $J=2 \rightarrow 1$ & 220398.686 & 12 & 53 & 0.15 \\
${ }^{13} \mathrm{CO}$ & $J=1 \rightarrow 0$ & 110201.370 & 24 & 27 & $0.90^{a}$ \\
${ }^{13} \mathrm{CO}$ & $J=2 \rightarrow 1$ & 220398.686 & 12 & 53 & $0.90^{a}$ \\
$\mathrm{C}^{18} \mathrm{O}$ & $J=1 \rightarrow 0$ & 109782.182 & 24 & 27 & 0.15 \\
$\mathrm{C}^{18} \mathrm{O}$ & $J=1 \rightarrow 0$ & 109782.182 & 24 & 27 & $0.90^{a}$ \\
\hline${ }^{3 \mathrm{rd}} \mathrm{setting}$ & & & & & \\
\hline $\mathrm{N}_{2} \mathrm{H}^{+}$ & $J=1 \rightarrow 0$ & $93173.764^{b}$ & 27 & 31 & 0.04 \\
$\mathrm{C}^{18} \mathrm{O}$ & $J=2 \rightarrow 1$ & 219560.358 & 12 & 53 & 0.05 \\
\hline
\end{tabular}

Notes. ${ }^{(a)}$ Raw value for on-the-fly mapping. ${ }^{(b)}$ Main hyperfine components, frequency from Pagani et al. (2009). 